\title{
Preprojective algebras and cluster algebras
}

\author{
Christof Geiss, Bernard Leclerc and Jan Schröer*
}

\begin{abstract}
We use the representation theory of preprojective algebras to construct and study certain cluster algebras related to semisimple algebraic groups.
\end{abstract}

Mathematics Subject Classification (2000). Primary 16G20; Secondary 14M15, 16D90, 16G70, 17B10, 20G05, 20G20, 20G42.

Keywords. preprojective algebra, cluster algebra, flag variety, rigid module, mutation, Frobenius category, semicanonical basis

\section{Introduction}

Cluster algebras were invented by Fomin and Zelevinsky in 2001 [10]. One of the main motivations for introducing this new class of commutative algebras was to provide a combinatorial and algebraic framework for studying the canonical bases of quantum groups introduced by Lusztig and Kashiwara [26, 22] and the notion of total positivity for semisimple algebraic groups developed by Lusztig [29].

A first attempt to understand cluster algebras in terms of the representation theory of quivers was done by Marsh, Reineke, and Zelevinsky [33], using a category of decorated representations. This was quickly followed by the seminal paper of Buan, Marsh, Reiten, Reineke, and Todorov [4] who introduced a new family of triangulated categories attached to hereditary algebras, called cluster categories, and showed that the combinatorics of cluster mutations arises in the tilting theory of these cluster categories. This yielded a categorification of a large family of cluster algebras: the acyclic cluster algebras.

In this review, we will explain a different but somewhat parallel development aimed at giving a representation-theoretic treatment for another class of cluster algebras, namely those discovered by Berenstein, Fomin and Zelevinsky [3] in relation with their series of works on total positivity and the geometry of double Bruhat cells in semisimple groups. Instead of the cluster categories we have used the module categories of the preprojective algebras corresponding to these semisimple groups, and more generally certain Frobenius subcategories of these module categories. This allowed us to prove that the cluster monomials of the cluster algebras we consider belong to the dual of Lusztig's semicanonical basis, and in particular are linearly independent [14]. It also enabled us to introduce new cluster algebra structures on the coordinate rings of partial flag varieties [17].

\footnotetext{
${ }^{*}$ We are grateful to A. Skowroński and the ICRA committee for encouraging us to prepare this survey for the ICRA XII book.
} 


\section{Total positivity, canonical bases and cluster al- gebras}

Before reviewing our construction, we would like to illustrate by means of some simple examples why total positivity, canonical bases and cluster algebras are intimately connected.

Lusztig has defined the totally positive part $X_{>0}$ for several classes of complex algebraic varieties $X$ attached to a semisimple algebraic group $G$. The definition uses the theory of canonical bases for irreducible $G$-modules. It is not our intention in this survey to explain this definition, neither to recall the construction of canonical bases (for excellent reviews of these topics, we refer the reader to $[28,31]$ ). Instead of this, we shall present a few examples for which both the totally positive varieties and the canonical bases can be described in an explicit and elementary way. We shall then see that a cluster algebra structure on the coordinate ring naturally arises from this description.

Our first example is trivial, but nevertheless useful to get started. Let $V=\mathbb{C}^{2 n}$ be an even dimensional vector space with natural coordinates $\left(y_{1}, \ldots, y_{2 n}\right)$. Let $X=\mathbb{P}(V)=\mathbb{P}^{2 n-1}$ be the corresponding projective space. In this case our group $G$ is $S L(V)$. The totally positive part of $V$ is simply the orthant

$$
V_{>0}=\left\{v \in V \mid y_{1}(v)>0, \ldots, y_{2 n}(v)>0\right\},
$$

and the totally positive part of $X$ is the subset of $X$ consisting of points having a system of homogeneous coordinates $\left(y_{1}: \cdots: y_{2 n}\right)$ with all $y_{i}$ positive. The coordinate ring of $V$ (or the homogeneous coordinate ring of $X$ ) is $R=\mathbb{C}\left[y_{1}, \ldots, y_{2 n}\right]$. It has a natural $\mathbb{C}$-basis given by all monomials in the $y_{i}$ 's. The natural action of $G$ on $V$ makes $R$ into a linear representation of $G$, which decomposes into irreducible representations as

$$
R=\bigoplus_{k \geq 0} R_{k}
$$

where $R_{k} \cong S^{k}\left(V^{*}\right)$ is the degree $k$ homogeneous part of $R$. For every irreducible representation of $G$, Lusztig and Kashiwara have introduced a canonical basis and a dual canonical basis (also called lower global basis and upper global basis by Kashiwara). It is not difficult to check that, in the case of the simple representations $R_{k}$, the dual canonical basis coincides with the basis of monomials in the $y_{i}$ 's of total degree $k$.

We now pass to a more interesting example. Consider the nondegenerate quadratic form on $V$ given by

$$
q\left(y_{1}, \ldots, y_{2 n}\right)=\sum_{i=1}^{n}(-1)^{i-1} y_{i} y_{2 n+1-i} .
$$

Let

$$
\mathcal{C}=\{v \in V \mid q(v)=0\}
$$

be its isotropic cone and $\mathcal{Q}=\mathbb{P}(\mathcal{C})$ the corresponding smooth quadric in $X=\mathbb{P}(V)$. The quadric $\mathcal{Q}$ can be seen as a partial flag variety for the special orthogonal group 
$H$ attached to the form $q$, and so it has, following Lusztig [30], a well-defined totally positive part. Let us try to guess what is $\mathcal{Q}_{>0}$, or equivalently what is $\mathcal{C}_{>0}$.

It seems natural to require that $\mathcal{C}_{>0}$ is contained in $V_{>0}$. But this is not enough, and in general $\mathcal{C}_{>0}$ is going to be a proper subset of $\mathcal{C} \cap V_{>0}$. To see this, we may use the known fact that the totally positive part of a variety of dimension $k$ is homeomorphic to $\mathbb{R}_{>0}^{k}$, hence we might expect that it is described by a system of $k$ inequalities. However, $\mathcal{C} \cap V_{>0}$ is the subset of $\mathcal{C}$ given by the $2 n$ inequalities $y_{i}>0$, and since $\mathcal{C}$ has dimension $2 n-1$, we would like to define $\mathcal{C}_{>0}$ by a system of only $2 n-1$ inequalities. In other words, there should exist a system of $2 n-1$ functions $\left(f_{1}, \ldots, f_{2 n-1}\right)$ on $\mathcal{C}$ such that

$$
\mathcal{C}_{>0}=\left\{v \in \mathcal{C} \mid f_{1}(v)>0, \ldots, f_{2 n-1}(v)>0\right\} .
$$

Such a system $\left(f_{1}, \ldots, f_{2 n-1}\right)$ is called a positive coordinate system.

So we are looking for a "natural" set of $2 n-1$ functions $\left(f_{1}, \ldots, f_{2 n-1}\right)$ such that the positivity of $f_{1}(v), \ldots, f_{2 n-1}(v)$ implies the positivity of $y_{1}(v), \ldots, y_{2 n}(v)$. Let us try this idea in the case $n=3$. We have

$$
q\left(y_{1}, \ldots, y_{6}\right)=y_{1} y_{6}-y_{2} y_{5}+y_{3} y_{4}
$$

On $\mathcal{C} \cap V_{>0}$ we therefore have the relation

$$
y_{5}=\frac{y_{1} y_{6}+y_{3} y_{4}}{y_{2}}
$$

Hence the positivity of the 5 coordinates in the right-hand side implies the positivity of $y_{5}$, that is, the defining equation of $\mathcal{C}$ allows to eliminate the inequality $y_{5}>0$ from the 6 defining inequalities of $\mathcal{C} \cap V_{>0}$. So we could take

$$
\left(y_{1}, y_{6}, y_{3}, y_{4}, y_{2}\right)
$$

as a positive coordinate system. Note that $y_{2}$ and $y_{5}$ play the same role and are exchangeable: we could also take $\left(y_{1}, y_{6}, y_{3}, y_{4}, y_{5}\right)$. This would define the same subset $\mathcal{C}_{>0}$, which in this case is simply $\mathcal{C} \cap V_{>0}$.

Already in the case $n=4$ the same trick no longer works. Indeed, the defining equation of $\mathcal{C}$ is now $y_{1} y_{8}-y_{2} y_{7}+y_{3} y_{6}-y_{4} y_{5}=0$, which does not allow us to express any of the $y_{i}$ 's as a subtraction-free expression in terms of the 7 remaining ones. To overcome this problem, we introduce a new quadratic function

$$
p=y_{3} y_{6}-y_{4} y_{5}=y_{2} y_{7}-y_{1} y_{8}
$$

on $\mathcal{C}$. On $\mathcal{C} \cap V_{>0}$ we then have

$$
y_{7}=\frac{y_{1} y_{8}+p}{y_{2}}, \quad y_{6}=\frac{y_{4} y_{5}+p}{y_{3}}
$$

and this leads us to take

$$
\left(y_{1}, y_{8}, y_{4}, y_{5}, p, y_{2}, y_{3}\right)
$$


as a positive coordinate system. Again, $y_{2}$ and $y_{7}$ are exchangeable, as are $y_{3}$ and $y_{6}$. So we would obtain the same subset $\mathcal{C}_{>0}$ by using, instead of (2), each of the 3 alternative systems of coordinates

$$
\left(y_{1}, y_{8}, y_{4}, y_{5}, p, y_{7}, y_{3}\right), \quad\left(y_{1}, y_{8}, y_{4}, y_{5}, p, y_{2}, y_{6}\right), \quad\left(y_{1}, y_{8}, y_{4}, y_{5}, p, y_{7}, y_{6}\right)
$$

Note that in this case our candidate for $\mathcal{C}_{>0}$ is a proper subset of $\mathcal{C} \cap V_{>0}$, since the positivity of $p$ does not follow from the positivity of the $y_{i}$ 's.

It turns out that this naive candidate coincides with the totally positive part of $\mathcal{C}$ defined by Lusztig. To explain this, let us consider the coordinate ring

$$
A=\mathbb{C}\left[y_{1}, \ldots, y_{8}\right] /\left(y_{1} y_{8}-y_{2} y_{7}+y_{3} y_{6}-y_{4} y_{5}\right)
$$

of $\mathcal{C}$, or in other words the homogeneous coordinate ring of the quadric $\mathcal{Q}$. As before, $A$ is in a natural way a representation of the special orthogonal group $H$, and the homogeneous components $A_{k}(k \geq 0)$ coincide with the irreducible direct summands of this representation. Hence by putting together the dual canonical bases of all summands $A_{k}$, we get a dual canonical basis of $A$. We claim that in this easy situation, the dual canonical basis can be explicitly computed and has the following simple description. Namely, the dual canonical basis of $A_{k}$ consists of all the degree $k$ monomials in $y_{1}, \ldots, y_{8}, p$ containing only variables of one of the 4 coordinate systems displayed in $(2),(3)$. Here, $y_{1}, \ldots, y_{8}$ have degree 1 and $p$ has degree 2.

For example, the dual canonical basis of $A_{1} \cong V^{*}$ is $\left\{y_{1}, y_{2}, y_{3}, y_{4}, y_{5}, y_{6}, y_{7}, y_{8}\right\}$, and the dual canonical basis of $A_{2}$ consists of $p$ and of all the degree 2 monomials in the $y_{i}$ 's except $y_{2} y_{7}$ and $y_{3} y_{6}$.

Now, Lusztig has shown [30, Prop. 3.2, Th. 3.4] that $\mathcal{C}_{>0}$ has the following characterization: it consists of all elements $v$ of $\mathbb{C}^{2 n}$ such that, for every element $b$ of the dual canonical basis of $A_{k}$ and for every $k$, one has $b(v)>0$. Because of the monomial description of the dual canonical basis, we see that this agrees with our naive definition of $\mathcal{C}_{>0}$.

Exercise 2.1. Guess in a similar way what is the definition of $\mathcal{C}_{>0}$ for $n \geq 4$.

Answer. For $s=1,2, \ldots, n-3$, put

$$
p_{s}=\sum_{k=1}^{s+1}(-1)^{s+1-k} y_{k} y_{2 n+1-k} .
$$

Then $\mathcal{C}_{>0}$ is the subset of $\mathcal{C}$ given by the following $n+1$ inequalities

$$
y_{1}>0, \quad y_{n}>0, \quad y_{n+1}>0, \quad y_{2 n}>0, \quad p_{s}>0,(s=1, \ldots, n-3),
$$

together with one (it does not matter which one) of the two inequalities

$$
y_{k}>0, \quad y_{2 n+1-k}>0,
$$

for each $k=2,3, \ldots, n-1$. 
Thus for every $n, \mathcal{C}_{>0}$ can be described as in (1) by a positive coordinate system, and there are $2^{n-2}$ different but equivalent such systems. In fact, one can also check that the dual canonical basis of the coordinate ring of $\mathcal{C}$ consists of all monomials in the $y_{i}$ 's and $p_{s}$ 's supported on one of these $2^{n-2}$ coordinate systems.

The definition of a cluster algebra will be recalled in Section 14 below. A reader already familiar with it will immediately recognize an obvious cluster algebra structure on the coordinate ring of $\mathcal{C}$ emerging from this discussion. Its $2(n-2)$ cluster variables are

$$
y_{k}, \quad y_{2 n+1-k}, \quad(k=2,3, \ldots, n-1) .
$$

Its coefficient ring is generated by

$$
y_{1}, y_{n}, y_{n+1}, y_{2 n}, p_{s}, \quad(s=1, \ldots, n-3)
$$

Its clusters are the $2^{n-2}$ possible choices of $2 n-1$ of these functions forming a positive coordinate system. Its cluster monomials are all the monomials supported on a single cluster, and its exchange relations are

$$
y_{k} y_{2 n+1-k}= \begin{cases}p_{1}+y_{1} y_{2 n} & \text { if } k=2 \\ p_{k-1}+p_{k-2} & \text { if } 3 \leq k \leq n-2 \\ y_{n} y_{n+1}+p_{n-3} & \text { if } k=n-1\end{cases}
$$

This is a cluster algebra of finite type (it has finitely many cluster variables). Its type, according to Fomin and Zelevinsky's classification [11] is $A_{1}^{n-2}$.

To summarize, the cone $\mathcal{C}$ and the corresponding quadric $\mathcal{Q}$ are examples of algebraic varieties for which Lusztig has described a natural totally positive subset $\mathcal{C}_{>0}$ or $\mathcal{Q}_{>0}$. What we have found is that their coordinate ring is endowed with the structure of a cluster algebra such that

(1) each cluster gives rise to a positive coordinate system;

(2) the dual canonical basis of the coordinate ring coincides with the set of cluster monomials.

This is the prototype of what one would like to do for each variety $X$ having a totally positive part $X_{>0}$ in Lusztig's sense. But in general, things become more complicated. First, the cluster algebra structure, when it is known, is usually wellhidden, and its description requires a lot of difficult (but beautiful) combinatorics. As an example, one may consult the paper of Scott [34] and in particular the cluster structures of the Grassmannians $\operatorname{Gr}(3,6), \operatorname{Gr}(3,7)$ and $\operatorname{Gr}(3,8)$. Secondly, these cluster algebras are generally of infinite type so one cannot hope for a closed and finite description as in the above example. This is not too bad if one is mainly interested in total positivity, since one may not necessarily need to know all positive coordinate systems on $X$. But it becomes a challenging issue if one aims at a monomial description of the dual canonical basis of the coordinate ring, because that would likely involve infinitely many families of monomials. In fact 
such a monomial description may not even be possible, since, as shown in [25], there may exist elements of the dual canonical basis whose square does not belong to the basis. In any case, even if the cluster structure is known, more work is certainly needed to obtain from it a full description of the dual canonical basis. Finally, there is no universal recipe for getting a cluster structure on the coordinate ring. Actually, the existence of such a structure is not guaranteed by any general theorem, so it often seems kind of miraculous when it eventually comes out of some complicated calculations.

The aim of this review is to explain some recent progress made in these directions by means of the representation theory of preprojective algebras. We will choose as our main example the partial flag varieties $X$ attached to a simple algebraic group $G$ of type $A, D, E$. Thus $X$ is a homogeneous space $G / P$, where $P$ is a parabolic subgroup of $G$. To $G$ one can attach the preprojective algebra $\Lambda$ with the same Dynkin type. To $P$ (or rather to its conjugacy class) one can attach a certain injective $\Lambda$-module $Q$, and the subcategory $\operatorname{Sub} Q$ of the module category $\bmod \Lambda$ cogenerated by $Q$. We will show that $\operatorname{Sub} Q$ can be regarded as a categorification of the multihomogeneous coordinate ring of $X$, and that the rigid modules in $\operatorname{Sub} Q$ give rise to a cluster structure on this ring. In particular, this yields a uniform recipe for producing explicit cluster structures, many of which were first discovered in this way. The cluster structure is of finite type when $\operatorname{Sub} Q$ has finite representation type, and in these exceptional cases, the Auslander-Reiten quiver of $Q$ is quite helpful for understanding the ensuing combinatorics. Finally, this approach allows to show that the cluster monomials of these algebras belong to the dual of Lusztig's semicanonical basis. Unfortunately, the relation between the semicanonical and the canonical basis is a subtle question (see [12]). Nevertheless, as predicted by the general conjecture of Fomin and Zelevinsky [10, p.498], we believe that the cluster monomials also belong to the dual canonical basis, that is, we conjecture that they lie in the intersection of the dual canonical basis and the dual semicanonical basis (see below $§ 19$ ).

\section{Preprojective algebras}

We start with definitions and basic results about preprojective algebras of Dynkin type.

Let $\Delta$ be a Dynkin diagram of type $A, D$ or $E$. We denote by $I$ the set of vertices and by $n$ its cardinality. Let $\bar{Q}$ be the quiver obtained from $\Delta$ by replacing each edge, between $i$ and $j$ say, by a pair of opposite arrows $a: i \longrightarrow j$ and $a^{*}: j \longrightarrow i$. Let $\mathbb{C} \bar{Q}$ denote the path algebra of $\bar{Q}$ over $\mathbb{C}$. We can form the following quadratic element in $\mathbb{C} \bar{Q}$,

$$
c=\sum\left(a^{*} a-a a^{*}\right),
$$

where the sum is over all edges of $\Delta$. Let $(c)$ be the two-sided ideal generated by $c$. Following Gelfand and Ponomarev [20], we define the preprojective algebra

$$
\Lambda:=\mathbb{C} \bar{Q} /(c) \text {. }
$$


It is well-known that $\Lambda$ is a finite-dimensional selfinjective algebra. It has finite representation type if $\Delta$ has type $A_{n}(n \leq 4)$, tame type if $\Delta$ has type $A_{5}$ or $D_{4}$, and wild type in all other cases (see [7]).

We denote by $S_{i}(i \in I)$ the simple $\Lambda$-modules, and by $Q_{i}(i \in I)$ their injective envelopes.

Example 3.1. Let $\Lambda$ be of type $D_{4}$. We label the 3 external nodes of the Dynkin diagram of type $D_{4}$ by $1,2,4$, and the central node by 3 . With this convention, the socle filtration of $Q_{4}$ is

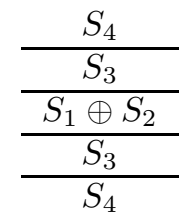

and the socle filtration of $Q_{3}$ is

$\frac{\frac{S_{3}}{S_{1} \oplus S_{2} \oplus S_{4}}}{\frac{S_{3} \oplus S_{3}}{S_{1} \oplus S_{2} \oplus S_{4}}}$

The structure of $Q_{1}$ and $Q_{2}$ can be obtained from that of $Q_{4}$ by applying the order 3 diagram automorphism $1 \mapsto 2 \mapsto 4 \mapsto 1$.

One important property of the module category $\bmod \Lambda$ is the following Extsymmetry. Let $D$ denote duality with respect to the field $\mathbb{C}$. We have

$$
\operatorname{Ext}_{\Lambda}^{1}(M, N) \cong D \operatorname{Ext}_{\Lambda}^{1}(N, M), \quad(M, N \in \bmod \Lambda),
$$

and this isomorphism is functorial with respect to $M$ and $N$ (see [16]).

\section{Regular functions on maximal unipotent sub- groups}

We turn now to semisimple algebraic groups. For unexplained terminology, the reader can consult standard references, e.g. [6], [8], [21].

Let $G$ be a simply connected simple complex algebraic group with the same Dynkin diagram $\Delta$ as $\Lambda$. Let $N$ be a fixed maximal unipotent subgroup of $G$. If $G=\mathrm{SL}(n+1, \mathbb{C})$, we can take $N$ to be the subgroup of upper unitriangular matrices. In general $N$ is less easy to describe. To perform concrete calculations, one can use the one-parameter subgroups $x_{i}(t)(i \in I, t \in \mathbb{C})$ associated with the simple roots, which form a distinguished set of generators of $N$.

Example 4.1. In type $A_{n}$, if $N$ is the subgroup of upper unitriangular matrices of $\mathrm{SL}(n+1, \mathbb{C})$, we have $x_{i}(t)=I+t E_{i, i+1}$, where $I$ is the identity matrix and $E_{i j}$ the matrix unit with a unique nonzero entry equal to 1 in row $i$ and column $j$. 
Example 4.2. In type $D_{n}, N$ can be identified with the subgroup of the group of upper unitriangular matrices of $\operatorname{SL}(2 n, \mathbb{C})$, generated by

$$
x_{i}(t)= \begin{cases}I+t\left(E_{n-i+1, n-i+2}+E_{n+i-1, n+i}\right) & \text { if } 2 \leq i \leq n \\ I+t\left(E_{n-1, n+1}+E_{n, n+2}\right) & \text { if } i=1\end{cases}
$$

Thus in type $D_{4}$ we can take for $N$ the subgroup of $\mathrm{SL}(8, \mathbb{C})$ generated by

$$
\begin{aligned}
& x_{1}(t)=\left(\begin{array}{cccccccc}
1 & 0 & 0 & 0 & 0 & 0 & 0 & 0 \\
0 & 1 & 0 & 0 & 0 & 0 & 0 & 0 \\
0 & 0 & 1 & 0 & t & 0 & 0 & 0 \\
0 & 0 & 0 & 1 & 0 & t & 0 & 0 \\
0 & 0 & 0 & 0 & 1 & 0 & 0 & 0 \\
0 & 0 & 0 & 0 & 0 & 1 & 0 & 0 \\
0 & 0 & 0 & 0 & 0 & 0 & 1 & 0 \\
0 & 0 & 0 & 0 & 0 & 0 & 0 & 1
\end{array}\right), \quad x_{2}(t)=\left(\begin{array}{cccccccc}
1 & 0 & 0 & 0 & 0 & 0 & 0 & 0 \\
0 & 1 & 0 & 0 & 0 & 0 & 0 & 0 \\
0 & 0 & 1 & t & 0 & 0 & 0 & 0 \\
0 & 0 & 0 & 1 & 0 & 0 & 0 & 0 \\
0 & 0 & 0 & 0 & 1 & t & 0 & 0 \\
0 & 0 & 0 & 0 & 0 & 1 & 0 & 0 \\
0 & 0 & 0 & 0 & 0 & 0 & 1 & 0 \\
0 & 0 & 0 & 0 & 0 & 0 & 0 & 1
\end{array}\right) \text {, } \\
& x_{3}(t)=\left(\begin{array}{cccccccc}
1 & 0 & 0 & 0 & 0 & 0 & 0 & 0 \\
0 & 1 & t & 0 & 0 & 0 & 0 & 0 \\
0 & 0 & 1 & 0 & 0 & 0 & 0 & 0 \\
0 & 0 & 0 & 1 & 0 & 0 & 0 & 0 \\
0 & 0 & 0 & 0 & 1 & 0 & 0 & 0 \\
0 & 0 & 0 & 0 & 0 & 1 & t & 0 \\
0 & 0 & 0 & 0 & 0 & 0 & 1 & 0 \\
0 & 0 & 0 & 0 & 0 & 0 & 0 & 1
\end{array}\right), \quad x_{4}(t)=\left(\begin{array}{cccccccc}
1 & t & 0 & 0 & 0 & 0 & 0 & 0 \\
0 & 1 & 0 & 0 & 0 & 0 & 0 & 0 \\
0 & 0 & 1 & 0 & 0 & 0 & 0 & 0 \\
0 & 0 & 0 & 1 & 0 & 0 & 0 & 0 \\
0 & 0 & 0 & 0 & 1 & 0 & 0 & 0 \\
0 & 0 & 0 & 0 & 0 & 1 & 0 & 0 \\
0 & 0 & 0 & 0 & 0 & 0 & 1 & t \\
0 & 0 & 0 & 0 & 0 & 0 & 0 & 1
\end{array}\right) .
\end{aligned}
$$

As an algebraic variety, $N$ is isomorphic to an affine space of complex dimension the number $r$ of positive roots of $\Delta$. Hence its coordinate ring $\mathbb{C}[N]$ is isomorphic to a polynomial ring in $r$ variables. For example in type $A_{n}$ if $N$ is the group of unitriangular matrices, each matrix entry $n_{i j}(1 \leq i<j \leq n+1)$ is a regular function on $N$ and $\mathbb{C}[N]$ is the ring of polynomials in the $n(n+1) / 2$ variables $n_{i j}$.

In the general case, the most convenient way of specifying a regular function $f \in \mathbb{C}[N]$ is to describe its evaluation $f\left(x_{i_{1}}\left(t_{1}\right) \cdots x_{i_{k}}\left(t_{k}\right)\right)$ at an arbitrary product of elements of the one-parameter subgroups. In fact one can restrict to certain special products. Namely, let $W$ denote the Weyl group of $G$ and $s_{i}(i \in I)$ its Coxeter generators. Let $w_{0}$ be the longest element of $W$ and let $w_{0}=s_{i_{1}} \cdots s_{i_{r}}$ be a reduced decomposition. Then it is well-known that the image of the map

$$
\left(t_{1}, \ldots, t_{r}\right) \in \mathbb{C}^{r} \mapsto x_{i_{1}}\left(t_{1}\right) \cdots x_{i_{r}}\left(t_{r}\right) \in N
$$

is a dense subset of $N$. It follows that a polynomial function $f \in \mathbb{C}[N]$ is completely determined by its values on this subset.

\section{A map from $\bmod \Lambda$ to $\mathbb{C}[N]$}

In [27, Section 12], Lusztig has given a geometric construction of the enveloping algebra $U(\mathfrak{n})$ of the Lie algebra of $N$. It is very similar to Ringel's realization of 
$U(\mathfrak{n})$ as the Hall algebra of $\bmod \left(\mathbb{F}_{q} Q\right)$ "specialized at $q=1$ ". Here $Q$ denotes any quiver obtained by orienting the edges of the Dynkin diagram $\Delta$.

There are two main differences between Ringel's and Lusztig's constructions. First, in Lusztig's approach one works directly at $q=1$ by replacing the counting measure for varieties over finite fields by the Euler characteristic measure for constructible subsets of complex algebraic varieties. The second difference is that one replaces the module varieties of $Q$ by the module varieties of $\Lambda$, in order to obtain a construction independent of the choice of an orientation of $\Delta$.

As a result, one gets a model of $U(\mathfrak{n})$ in which the homogeneous piece $U(\mathfrak{n})_{\mathbf{d}}$ of multidegree $\mathbf{d}=\left(d_{i}\right) \in \mathbb{N}^{I}$ is realized as a certain vector space of complex-valued constructible functions on the variety $\Lambda_{\mathbf{d}}$ of $\Lambda$-modules with dimension vector $\mathbf{d}$. It follows that to every $M \in \bmod \Lambda$ of dimension vector $\mathbf{d}$, one can attach a natural element of the dual space $U(\mathfrak{n})_{\mathbf{d}}^{*}$, namely the linear form $\delta_{M}$ mapping a constructible function $\psi \in U(\mathfrak{n})_{\mathbf{d}}$ to its evaluation at $M$ (by regarding $M$ as a point of $\Lambda_{\mathbf{d}}$ ). Let

$$
U(\mathfrak{n})_{\mathrm{gr}}^{*}:=\bigoplus_{\mathbf{d} \in \mathbb{N}^{I}} U(\mathfrak{n})_{\mathbf{d}}^{*}
$$

be the graded dual of $U(\mathfrak{n})$ endowed with the dual Hopf structure. The following result is well-known to the experts, but we were unable to find a convenient reference. We include a sketch of proof for the convenience of the reader.

Proposition 5.1. $U(\mathfrak{n})_{\mathrm{gr}}^{*}$ is isomorphic, as a Hopf algebra, to $\mathbb{C}[N]$.

Proof. (Sketch.) $H=U(\mathfrak{n})_{\mathrm{gr}}^{*}$ is a commutative Hopf algebra, and therefore it can be regarded as the coordinate ring of the affine algebraic group $\operatorname{Hom}_{\text {alg }}(H, \mathbb{C})$ of algebra homomorphisms from $H$ to $\mathbb{C}$, or equivalently as the coordinate ring of the group $G\left(H^{\circ}\right)$ of all group-like elements in the dual Hopf algebra $H^{\circ}$ (see e.g. $[1, \S 3.4])$. Note that $H$ being the graded dual of $U(\mathfrak{n})$, the dual $H^{*}$ of $H$ is the completion $\widehat{U(\mathfrak{n})}$ of $U(\mathfrak{n})$ with respect to its grading. A simple calculation shows that for every $e \in \mathfrak{n}$ the $\operatorname{exponential} \exp (e)=\sum_{k \geq 0} e^{k} / k ! \in \widehat{U(\mathfrak{n})}$ is a group-like element in $H^{\circ}$. Let $e_{i}(i \in I)$ be the Chevalley generators of $\mathfrak{n}$. Then the map $x_{i}(t) \mapsto \exp \left(t e_{i}\right)(i \in I)$ extends to a homomorphism from $N$ to $G\left(H^{\circ}\right)$. One can check that this is an isomorphism using the fact that $H$ is a polynomial algebra in $r$ variables. This induces the claimed Hopf algebra isomorphism from $H$ to $\mathbb{C}[N]$.

Let $\iota: U(\mathfrak{n})_{\mathrm{gr}}^{*} \rightarrow \mathbb{C}[N]$ denote this isomorphism. Let $\omega$ denote the automorphism of $\mathbb{C}[N]$ described in $[15, \S 1.7]$. It anti-commutes with the comultiplication, and the corresponding anti-automorphism of $N$ leaves invariant the one-parameter subgroups $x_{i}(t)$. In other words, for $f \in \mathbb{C}[N]$ we have

$$
(\omega f)\left(x_{i_{1}}\left(t_{1}\right) \cdots x_{i_{k}}\left(t_{k}\right)\right)=f\left(x_{i_{k}}\left(t_{k}\right) \cdots x_{i_{1}}\left(t_{1}\right)\right), \quad\left(i_{1}, \ldots i_{k} \in I, t_{1}, \ldots, t_{k} \in \mathbb{C}\right) .
$$

Define $\varphi_{M}=\omega \circ \iota\left(\delta_{M}\right)$. We have thus obtained a map $M \mapsto \varphi_{M}$ from $\bmod \Lambda$ to $\mathbb{C}[N]$. Let us describe it more explicitly. 
Consider a composition series

$$
\mathfrak{f}=\left(\{0\}=M_{0} \subset M_{1} \subset \cdots \subset M_{d}=M\right)
$$

of $M$ with simple factors $M_{k} / M_{k-1} \cong S_{i_{k}}$. We call $\mathbf{i}:=\left(i_{1}, \ldots, i_{d}\right)$ the type of $\mathfrak{f}$. Let $\Phi_{\mathbf{i}, M}$ denote the subset of the flag variety of $M$ (regarded as a $\mathbb{C}$-vector space) consisting of all flags which are in fact composition series of $M$ (regarded as a $\Lambda$-module) of type $\mathbf{i}$. This is a closed subset of the flag variety, hence a projective variety. We denote by $\chi_{\mathbf{i}, M}=\chi\left(\Phi_{\mathbf{i}, M}\right) \in \mathbb{Z}$ its Euler characteristic. By unwinding Lusztig's construction of $U(\mathfrak{n})$, dualizing it, and going through the above isomorphisms, one gets the following formula for $\varphi_{M}$.

Proposition 5.2. For every $\mathbf{i}=\left(i_{1}, \ldots, i_{k}\right) \in I^{k}$ we have

$$
\varphi_{M}\left(x_{i_{1}}\left(t_{1}\right) \cdots x_{i_{k}}\left(t_{k}\right)\right)=\sum_{\mathbf{a} \in \mathbb{N}^{k}} \chi_{\mathbf{i}^{\mathbf{a}}, M} \frac{t_{1}^{a_{1}} \cdots t_{k}^{a_{k}}}{a_{1} ! \cdots a_{k} !}
$$

where we use the short-hand notation $\mathbf{i}^{\mathbf{a}}=(\underbrace{i_{1}, \ldots, i_{1}}_{a_{1}}, \ldots, \underbrace{i_{k}, \ldots, i_{k}}_{a_{k}})$.

Proof. (Sketch.) Using the above embedding of $N$ in $\widehat{U(\mathfrak{n})}$, we have

$$
x_{i_{1}}\left(t_{1}\right) \cdots x_{i_{k}}\left(t_{k}\right)=\sum_{\mathbf{a} \in \mathbb{N}^{k}} \frac{t_{1}^{a_{1}} \cdots t_{k}^{a_{k}}}{a_{1} ! \cdots a_{k} !} e_{i_{1}}^{a_{1}} \cdots e_{i_{k}}^{a_{k}}
$$

as an element of $\widehat{U(\mathfrak{n})}$. Now, for a fixed $\mathbf{j}=\left(j_{1}, \ldots, j_{d}\right)$, consider the constructible function $\chi_{\mathbf{j}}: M \mapsto \chi_{\mathbf{j}, M}$ defined on $\Lambda_{\mathbf{d}}$, where $\mathbf{d}=\left(d_{i}\right)$ and $d_{i}$ is the number of $s$ 's such that $j_{s}=i$. In Lusztig's Lagrangian construction of $U(\mathfrak{n})$ [27], the functions $\chi_{\mathbf{j}}$ span the vector space $U(\mathfrak{n})_{\mathbf{d}}$. More precisely, $\chi_{\mathbf{j}}$ is identified with the monomial $e_{j_{d}} \cdots e_{j_{1}}$. By the definition of $\varphi_{M}$, we thus get

$$
\varphi_{M}\left(x_{i_{1}}\left(t_{1}\right) \cdots x_{i_{k}}\left(t_{k}\right)\right) \equiv \delta_{M}\left(\sum_{\mathbf{a} \in \mathbb{N}^{k}} \frac{t_{1}^{a_{1}} \cdots t_{k}^{a_{k}}}{a_{1} ! \cdots a_{k} !} \chi_{\mathbf{i}^{\mathbf{a}}}\right)=\sum_{\mathbf{a} \in \mathbb{N}^{k}} \chi_{\mathbf{i}^{\mathbf{a}}, M} \frac{t_{1}^{a_{1}} \cdots t_{k}^{a_{k}}}{a_{1} ! \cdots a_{k} !},
$$

as claimed. Note that the twist by $\omega$ and the twist $\chi_{j_{1}, \ldots, j_{d}} \equiv e_{j_{d}} \cdots e_{j_{1}}$ cancel each other.

Remark 5.3. In [14] we have denoted by $\varphi_{M}$ the function $\iota\left(\delta_{M}\right)$, without twisting by $\omega$ (in the definition of $\Phi_{\mathbf{i}, M}$ we were using descending flags $\mathfrak{f}$ instead of ascending ones). On the other hand, in $[13, \S 7]$ we have defined a left $U(\mathfrak{n})$-module structure on $U(\mathfrak{n})_{\mathrm{gr}}^{*}$. The twisting by $\omega$ is needed if we want this structure to agree with the usual left $U(\mathfrak{n})$-module structure on $\mathbb{C}[N]$ given by

$$
\left(e_{i} f\right)(x)=\left.\frac{d}{d t} f\left(x x_{i}(t)\right)\right|_{t=0}, \quad(f \in \mathbb{C}[N], x \in N) .
$$

This is the convention which we have taken in [17] and which we follow here. 
Example 5.4. In type $A_{2}$, we have $w_{0}=s_{1} s_{2} s_{1}$, hence every $f \in \mathbb{C}[N]$ is determined by its values at $x_{1}\left(t_{1}\right) x_{2}\left(t_{2}\right) x_{1}\left(t_{3}\right)$ for $\left(t_{1}, t_{2}, t_{3}\right) \in \mathbb{C}^{3}$. One calculates

$$
\begin{aligned}
x_{1}\left(t_{1}\right) x_{2}\left(t_{2}\right) x_{1}\left(t_{3}\right) & =\left(\begin{array}{ccc}
1 & t_{1} & 0 \\
0 & 1 & 0 \\
0 & 0 & 1
\end{array}\right)\left(\begin{array}{ccc}
1 & 0 & 0 \\
0 & 1 & t_{2} \\
0 & 0 & 1
\end{array}\right)\left(\begin{array}{ccc}
1 & t_{3} & 0 \\
0 & 1 & 0 \\
0 & 0 & 1
\end{array}\right) \\
& =\left(\begin{array}{ccc}
1 & t_{1}+t_{3} & t_{1} t_{2} \\
0 & 1 & t_{2} \\
0 & 0 & 1
\end{array}\right) .
\end{aligned}
$$

On the other hand, using the formula of Proposition 5.2 one gets easily

$$
\begin{aligned}
& \varphi_{S_{1}}\left(x_{1}\left(t_{1}\right) x_{2}\left(t_{2}\right) x_{1}\left(t_{3}\right)\right)=t_{1}+t_{3}, \\
& \varphi_{S_{2}}\left(x_{1}\left(t_{1}\right) x_{2}\left(t_{2}\right) x_{1}\left(t_{3}\right)\right)=t_{2}, \\
& \varphi_{Q_{1}}\left(x_{1}\left(t_{1}\right) x_{2}\left(t_{2}\right) x_{1}\left(t_{3}\right)\right)=t_{1} t_{2}, \\
& \varphi_{Q_{2}}\left(x_{1}\left(t_{1}\right) x_{2}\left(t_{2}\right) x_{1}\left(t_{3}\right)\right)=t_{2} t_{3} .
\end{aligned}
$$

It follows that, in terms of matrix entries, we have

$$
\varphi_{S_{1}}=n_{12}, \quad \varphi_{S_{2}}=n_{23}, \quad \varphi_{Q_{1}}=n_{13}, \quad \varphi_{Q_{2}}=\left|\begin{array}{cc}
n_{12} & n_{13} \\
1 & n_{23}
\end{array}\right| .
$$

Exercise 5.5. In type $A_{n}$, for $1 \leq i \leq j \leq n$, let $M_{[i, j]}$ denote the indecomposable $\Lambda$-module of dimension $j-i+1$ with socle $S_{i}$ and top $S_{j}$. ( $M_{[i, j]}$ is in fact uniserial.) Show that $\varphi_{M_{[i, j]}}=n_{i, j+1}$, the matrix entry on row $i$ and column $j+1$.

Exercise 5.6. In type $A_{n}$, show that $\varphi_{Q_{k}}$ is equal to the $k \times k$ minor of

$$
\left(\begin{array}{cccc}
1 & n_{12} & \ldots & n_{1, n+1} \\
0 & 1 & \ldots & n_{2, n+1} \\
\vdots & \vdots & \ddots & \vdots \\
0 & 0 & \ldots & 1
\end{array}\right)
$$

with row indices $1,2, \ldots, k$ and column indices $n-k+2, n-k+3, \ldots, n+1$.

More generally, show that for every submodule $M$ of $Q_{k}, \varphi_{M}$ is equal to a $k \times k$ minor with row indices $1,2, \ldots, k$, and that conversely, every nonzero $k \times k$ minor with row indices $1,2, \ldots, k$ is of the form $\varphi_{M}$ for a unique submodule $M$ of $Q_{k}$.

Exercise 5.7. In type $D_{4}$ a reduced decomposition of $w_{0}$ is for example

$$
w_{0}=s_{1} s_{2} s_{4} s_{3} s_{1} s_{2} s_{4} s_{3} s_{1} s_{2} s_{4} s_{3} .
$$

Using the realization of $N$ as a group of unitriangular $8 \times 8$ matrices given in Example 4.2, calculate (possibly with the help of your favorite computer algebra system) the product

$$
x=x_{1}\left(t_{1}\right) x_{2}\left(t_{2}\right) x_{4}\left(t_{3}\right) x_{3}\left(t_{4}\right) x_{1}\left(t_{5}\right) x_{2}\left(t_{6}\right) x_{4}\left(t_{7}\right) x_{3}\left(t_{8}\right) x_{1}\left(t_{9}\right) x_{2}\left(t_{10}\right) x_{4}\left(t_{11}\right) x_{3}\left(t_{12}\right) .
$$


Check that the first row of the matrix $x$ is equal to

$$
\begin{gathered}
{\left[1, \quad t_{3}+t_{7}+t_{11}, \quad t_{3} t_{4}+t_{3} t_{8}+t_{7} t_{8}+t_{3} t_{12}+t_{7} t_{12}+t_{11} t_{12},\right.} \\
t_{3} t_{4} t_{6}+t_{3} t_{4} t_{10}+t_{3} t_{8} t_{10}+t_{7} t_{8} t_{10}, \quad t_{3} t_{4} t_{5}+t_{3} t_{4} t_{9}+t_{3} t_{8} t_{9}+t_{7} t_{8} t_{9}, \\
t_{3} t_{4} t_{5} t_{6}+t_{3} t_{4} t_{5} t_{10}+t_{3} t_{4} t_{6} t_{9}+t_{3} t_{4} t_{9} t_{10}+t_{3} t_{8} t_{9} t_{10}+t_{7} t_{8} t_{9} t_{10}, \\
t_{3} t_{4} t_{5} t_{6} t_{8}+t_{3} t_{4} t_{5} t_{6} t_{12}+t_{3} t_{4} t_{6} t_{9} t_{12}+t_{3} t_{4} t_{5} t_{10} t_{12}+t_{3} t_{4} t_{9} t_{10} t_{12} \\
\left.\quad+t_{3} t_{8} t_{9} t_{10} t_{12}+t_{7} t_{8} t_{9} t_{10} t_{12}, \quad t_{3} t_{4} t_{5} t_{6} t_{8} t_{11}\right] .
\end{gathered}
$$

Check that the 8 entries on this row are equal to $\varphi_{M}(x)$ where $M$ runs over the 8 submodules of $Q_{4}$, including the zero and the full submodules (see Example 3.1).

Express in a similar way all the entries of $x$ as the evaluations at $x$ of functions $\varphi_{M}$ where $M$ runs over the subquotients of $Q_{4}$.

Investigate the relations between the $2 \times 2$ minors taken on the first 2 rows of $x$ and the values $\varphi_{M}(x)$ where $M$ is a submodule of $Q_{3}$.

\section{Multiplicative properties of $\varphi$}

In the geometric realization of $U(\mathfrak{n})$ given in [27], only the multiplication is constructed, or equivalently the comultiplication of $\mathbb{C}[N]$. For our purposes though, it is essential to study the multiplicative properties of the maps $\varphi_{M}$. The most important ones are

Theorem $6.1([12,16])$. Let $M, N \in \bmod \Lambda$. Then the following hold:

(1) $\varphi_{M} \varphi_{N}=\varphi_{M \oplus N}$.

(2) Assume that $\operatorname{dim} \operatorname{Ext}_{\Lambda}^{1}(M, N)=1$. Let

$$
0 \rightarrow M \rightarrow X \rightarrow N \rightarrow 0, \quad 0 \rightarrow N \rightarrow Y \rightarrow M \rightarrow 0,
$$

be two non-split short exact sequences (note that this determines $X$ and $Y$ uniquely up to isomorphism). Then $\varphi_{M} \varphi_{N}=\varphi_{X}+\varphi_{Y}$.

Note that in [16] a formula is proved which generalizes (2) to any pair $(M, N)$ of $\Lambda$-modules with $\operatorname{dim} \operatorname{Ext}_{\Lambda}^{1}(M, N)>0$. It involves all possible middle terms of non-split short exact sequences with end terms $M$ and $N$, weighted by certain Euler characteristics. It was inspired by a similar formula of Caldero and Keller in the framework of cluster categories [5]. We will not need this general multiplication formula here.

Example 6.2. Type $A_{2}$. Using the formulas of Example 5.4, one checks easily that

$$
\varphi_{S_{1}} \varphi_{S_{2}}=\varphi_{Q_{1}}+\varphi_{Q_{2}},
$$

in agreement with the short exact sequences

$$
0 \rightarrow S_{1} \rightarrow Q_{1} \rightarrow S_{2} \rightarrow 0, \quad 0 \rightarrow S_{2} \rightarrow Q_{2} \rightarrow S_{1} \rightarrow 0 .
$$


Exercise 6.3. Type $A_{3}$. Consider the following indecomposable $\Lambda$-modules defined unambiguously by means of their socle filtration:

$$
M=S_{2}, \quad N=\frac{S_{1} \oplus S_{3}}{S_{2}}, \quad X=\frac{S_{2}}{\frac{S_{1} \oplus S_{3}}{S_{2}}}=Q_{2}, \quad Y=\frac{S_{1}}{S_{2}}, \quad Z=\frac{S_{3}}{S_{2}} .
$$

Check that $\varphi_{M} \varphi_{N}=\varphi_{X}+\varphi_{Y \oplus Z}$. Using Exercise 5.6, show that this identity is nothing else than the classical Plücker relation

$$
[1,3] \times[2,4]=[1,2] \times[3,4]+[1,4] \times[2,3]
$$

between $2 \times 2$ minors of the matrix of coordinate functions

$$
\left(\begin{array}{cccc}
1 & n_{12} & n_{13} & n_{14} \\
0 & 1 & n_{23} & n_{24} \\
0 & 0 & 1 & n_{34} \\
0 & 0 & 0 & 1
\end{array}\right)
$$

where $[i, j]$ denotes the $2 \times 2$ minor on rows $(1,2)$ and columns $(i, j)$.

\section{The dual semicanonical basis}

The functions $\varphi_{M}(M \in \bmod \Lambda)$ satisfy many linear relations. For example if $\operatorname{dim} \operatorname{Ext}_{\Lambda}^{1}(M, N)=1$, combining (1) and (2) in Theorem 6.1 we get

$$
\varphi_{M \oplus N}=\varphi_{X}+\varphi_{Y}
$$

It is possible, though, to form bases of $\mathbb{C}[N]$ consisting of functions $\varphi_{M}$ where $M$ is taken in a certain restricted family of modules $M$. For example, let $Q$ be a fixed orientation of $\Delta$. Every $\mathbb{C} Q$-module can be regarded as a $\Lambda$-module in an obvious way. It is easy to check that

$$
\left\{\varphi_{M} \mid M \in \bmod (\mathbb{C} Q)\right\}
$$

is a $\mathbb{C}$-basis of $\mathbb{C}[N]$. In fact this is the dual of the PBW-basis of $U(\mathfrak{n})$ constructed from $Q$ by Ringel (see $[12, \S 5.9]$ ).

Unfortunately, this basis depends on the choice of the orientation $Q$. Using some geometry, one can obtain a more "canonical" basis of $\mathbb{C}[N]$. Let us fix a dimension vector $\mathbf{d} \in \mathbb{N}^{I}$ and regard the map $\varphi$ as a map from the module variety $\Lambda_{\mathbf{d}}$ to $\mathbb{C}[N]$. This is a constructible map, hence on every irreducible component of $\Lambda_{\mathbf{d}}$ there is a Zariski open set on which $M \mapsto \varphi_{M}$ is constant. Let us say that a module $M$ in this open set is generic. Then, dualizing Lusztig's construction in $[32]$, one gets

Theorem 7.1. $\left\{\varphi_{M} \mid M\right.$ is generic $\}$ is a basis of $\mathbb{C}[N]$. 
This is the dual of Lusztig's semicanonical basis of $U(\mathfrak{n})$. We shall call it the dual semicanonical basis of $\mathbb{C}[N]$. By construction it comes with a natural labelling by the union over all $\mathbf{d} \in \mathbb{N}^{I}$ of the sets of irreducible components of the varieties $\Lambda_{\mathbf{d}}$.

Important examples of generic modules are given by rigid modules. We say that a $\Lambda$-module $M$ is rigid if $\operatorname{Ext}_{\Lambda}^{1}(M, M)=0$, or equivalently if the orbit of $M$ in its module variety is open (see [14]).

Corollary 7.2. If $M$ is a rigid $\Lambda$-module then $\varphi_{M}$ belongs to the dual semicanonical basis of $\mathbb{C}[N]$.

The converse does not hold in general. More precisely every generic $\Lambda$-module is rigid if and only if $\Lambda$ has type $A_{n}(n \leq 4)$ (see [12]).

Example 7.3. In type $D_{4}$, there is a one-parameter family of indecomposable $\Lambda$-modules with socle series and radical series

$$
\frac{S_{3}}{\frac{S_{1} \oplus S_{2} \oplus S_{4}}{S_{3}}}
$$

These modules are generic, but they are not rigid. For example there is a selfextension with middle term $Q_{3}$.

\section{Dual Verma modules}

Let $\mathfrak{g}$ denote the Lie algebra of $G$ with its triangular decomposition $\mathfrak{g}=\mathfrak{n} \oplus \mathfrak{h} \oplus \mathfrak{n}_{-}$. Any $G$-module can also be regarded as a $\mathfrak{g}$-module. We shall denote by $L(\lambda)$ the irreducible finite-dimensional module with highest weight $\lambda$. It is conveniently constructed as the unique top factor of the infinite-dimensional Verma $\mathfrak{g}$-module $M(\lambda)$ (see e.g. $[6, \mathrm{I}, \S 3.2]$ ). As a $U\left(\mathfrak{n}_{-}\right)$-module, $M(\lambda)$ is naturally isomorphic to $U\left(\mathfrak{n}_{-}\right)$, hence we have a natural projection $U(\mathfrak{n}) \cong U\left(\mathfrak{n}_{-}\right) \cong M(\lambda) \rightarrow L(\lambda)$ for every weight $\lambda$. Dualizing and taking into account that $L(\lambda)$ is self-dual, we thus get an embedding $L(\lambda) \rightarrow M(\lambda)^{*} \cong \mathbb{C}[N]$. This embedding has a nice description in terms of the functions $\varphi_{M}$, as we shall now see.

Let $\lambda=\sum_{i \in I} a_{i} \varpi_{i}$ be the decomposition of $\lambda$ in terms of the fundamental weights $\varpi_{i}$. As $L(\lambda)$ is finite-dimensional, the $a_{i}$ 's are nonnegative integers. Set $Q_{\lambda}=\oplus_{i \in I} Q_{i}^{\oplus a_{i}}$, an injective $\Lambda$-module.

Theorem 8.1 ([13]). In the above identification of $M(\lambda)^{*}$ with $\mathbb{C}[N]$, the irreducible representation $L(\lambda)$ gets identified with the linear span of

$$
\left\{\varphi_{M} \mid M \text { is a submodule of } Q_{\lambda}\right\} .
$$

We refer to [13] for an explicit formula calculating the images of $\varphi_{M} \in L(\lambda)$ under the action of the Chevalley generators $e_{i}$ and $f_{i}$ of $\mathfrak{g}$. 
Example 8.2. In type $A_{n}$, consider the fundamental representation $L\left(\varpi_{k}\right)$. It is isomorphic to the natural representation of $\mathrm{SL}(n+1, \mathbb{C})$ in $\wedge^{k} \mathbb{C}^{n+1}$. Using Exercise 5.6, we recover via Theorem 8.1 that $L\left(\varpi_{k}\right)$ can be identified with the subspace of $\mathbb{C}[N]$ spanned by the $k \times k$-minors taken on the first $k$ rows of

$$
\left(\begin{array}{cccc}
1 & n_{12} & \ldots & n_{1, n+1} \\
0 & 1 & \ldots & n_{2, n+1} \\
\vdots & \vdots & \ddots & \vdots \\
0 & 0 & \cdots & 1
\end{array}\right)
$$

Example 8.3. In type $D_{4}$, consider the fundamental representation $L\left(\varpi_{4}\right)$. It is isomorphic to the defining representation of $G$ in $\mathbb{C}^{8}$. If we realize $N$ as a group of $8 \times 8$ unitriangular matrices as in Example 4.2 and use Exercise 5.7, we recover via Theorem 8.1 that $L\left(\varpi_{4}\right)$ can be identified with the subspace of $\mathbb{C}[N]$ spanned by the coordinate functions mapping an $8 \times 8$ matrix $x \in N$ to the entries of its first row.

\section{Parabolic subgroups and flag varieties}

Let us fix a proper subset $K$ of $I$. Denote by $y_{i}(t)(i \in I, t \in \mathbb{C})$ the one-parameter subgroups of $G$ attached to the negatives of the simple roots. Let $B$ be the Borel subgroup of $G$ containing $N$. The subgroup of $G$ generated by $B$ and the elements $y_{k}(t)(k \in K, t \in \mathbb{C})$ is called the standard parabolic subgroup attached to $K$. We shall denote it by $B_{K}$. In particular, $B_{\emptyset}=B$. It is known that every parabolic subgroup of $G$ is conjugate to a standard parabolic subgroup. The unipotent radical of $B_{K}$ will be denoted by $N_{K}$. In particular, $N_{\emptyset}=N$.

Example 9.1. Let $G=\operatorname{SL}(5, \mathbb{C})$, a group of type $A_{4}$. We choose for $B$ the subgroup of upper triangular matrices. Take $K=\{1,3,4\}$. Then $B_{K}$ and $N_{K}$ are the subgroups of $G$ with the following block form:

$$
B_{K}=\left(\begin{array}{ccccc}
* & * & * & * & * \\
* & * & * & * & * \\
0 & 0 & * & * & * \\
0 & 0 & * & * & * \\
0 & 0 & * & * & *
\end{array}\right), \quad N_{K}=\left(\begin{array}{ccccc}
1 & 0 & * & * & * \\
0 & 1 & * & * & * \\
0 & 0 & 1 & 0 & 0 \\
0 & 0 & 0 & 1 & 0 \\
0 & 0 & 0 & 0 & 1
\end{array}\right) .
$$

Geometrically, $N_{K}$ is an affine space. It can be identified with an open cell in the partial flag variety $B_{K}^{-} \backslash G$, where $B_{K}^{-}$is the opposite parabolic subgroup (defined as $B_{K}$ but switching the $x_{i}(t)$ 's and the $y_{i}(t)$ 's). More precisely, the restriction to $N_{K}$ of the natural projection $G \rightarrow B_{K}^{-} \backslash G$ is an open embedding. 
Example 9.2. Let us continue Example 9.1. We have

$$
B_{K}^{-}=\left(\begin{array}{ccccc}
* & * & 0 & 0 & 0 \\
* & * & 0 & 0 & 0 \\
* & * & * & * & * \\
* & * & * & * & * \\
* & * & * & * & *
\end{array}\right) .
$$

Let $\left(u_{1}, \ldots, u_{5}\right)$ be the standard basis of $\mathbb{C}^{5}$. We regard vectors of $\mathbb{C}^{5}$ as row vectors and we let $G$ act on the right on $\mathbb{C}^{5}$, so that the $k$ th row of the matrix $g$ is $u_{k} g$. Then $B_{K}^{-}$is the stabilizer of the 2 -space spanned by $u_{1}$ and $u_{2}$ for the induced transitive action of $G$ on the Grassmann variety of 2-planes of $\mathbb{C}^{5}$. Hence $B_{K}^{-} \backslash G$ is the Grassmannian $\operatorname{Gr}(2,5)$ of dimension 6 .

The unipotent subgroup $N_{K}$ can be identified with the open subset of $\operatorname{Gr}(2,5)$ consisting of all the 2-planes whose first Plücker coordinate $[1,2]$ does not vanish.

\section{Coordinate rings}

Let $J$ be the complement of $K$ in $I$. The partial flag variety $B_{K}^{-} \backslash G$ can be naturally embedded as a closed subset in the product of projective spaces

$$
\prod_{j \in J} \mathbb{P}\left(L\left(\varpi_{j}\right)\right)
$$

[24, p.123]. This is a generalization of the classical Plücker embedding of the Grassmannian $\operatorname{Gr}(k, n+1)$ in $\mathbb{P}\left(\wedge^{k} \mathbb{C}^{n+1}\right)=\mathbb{P}\left(L\left(\varpi_{k}\right)\right)$. We denote by $\mathbb{C}\left[B_{K}^{-} \backslash G\right]$ the multi-homogeneous coordinate ring of $B_{K}^{-} \backslash G$ coming from this embedding. Let $\Pi_{J} \cong \mathbb{N}^{J}$ denote the monoid of dominant integral weights of the form

$$
\lambda=\sum_{j \in J} a_{j} \varpi_{j}, \quad\left(a_{j} \in \mathbb{N}\right) .
$$

Then, $\mathbb{C}\left[B_{K}^{-} \backslash G\right]$ is a $\Pi_{J}$-graded ring with a natural $G$-module structure. The homogeneous component with multi-degree $\lambda \in \Pi_{J}$ is an irreducible $G$-module with highest weight $\lambda$. In other words, we have

$$
\mathbb{C}\left[B_{K}^{-} \backslash G\right]=\bigoplus_{\lambda \in \Pi_{J}} L(\lambda)
$$

Moreover, $\mathbb{C}\left[B_{K}^{-} \backslash G\right]$ is generated by its subspace $\bigoplus_{j \in J} L\left(\varpi_{j}\right)$.

In particular, $\mathbb{C}\left[B^{-} \backslash G\right]=\bigoplus_{\lambda \in \Pi} L(\lambda)$, where the sum is over the monoid $\Pi$ of all dominant integral weights of $G$. This is equal to the affine coordinate ring $\mathbb{C}\left[N^{-} \backslash G\right]$ of the multi-cone $N^{-} \backslash G$ over $B^{-} \backslash G$, that is, to the ring

$$
\mathbb{C}\left[N^{-} \backslash G\right]=\left\{f \in \mathbb{C}[G] \mid f(n g)=f(g), n \in N^{-}, g \in G\right\}
$$

of polynomial functions on $G$ invariant under $N^{-}$. We will identify $\mathbb{C}\left[B_{K}^{-} \backslash G\right]$ with the subalgebra of $\mathbb{C}\left[N^{-} \backslash G\right]$ generated by the homogeneous elements of degree $\varpi_{j}(j \in J)$. 
Example 10.1. We continue Example 9.2. The Plücker embedding of the Grassmannian $\operatorname{Gr}(2,5)$ consists in mapping the 2 -plane $V$ of $\mathbb{C}^{5}$ with basis $\left(v_{1}, v_{2}\right)$ to the line spanned by $v_{1} \wedge v_{2}$ in $\wedge^{2} \mathbb{C}^{5}$, which is isomorphic to $L\left(\varpi_{2}\right)$.

This induces an embedding of $\operatorname{Gr}(2,5)$ into $\mathbb{P}\left(L\left(\varpi_{2}\right)\right)$. The homogeneous coordinate ring for this embedding is isomorphic to the subring of $\mathbb{C}[G]$ generated by the functions $g \mapsto \Delta_{i j}(g)$, where $\Delta_{i j}(g)$ denotes the $2 \times 2$ minor of $g$ taken on columns $i, j$ and on the first two rows. The $\Delta_{i j}$ are called Plücker coordinates. As a $G$-module we have

$$
\mathbb{C}[\operatorname{Gr}(2,5)]=\bigoplus_{k \in \mathbb{N}} L\left(k \varpi_{2}\right),
$$

where the degree $k$ homogeneous component $L\left(k \varpi_{2}\right)$ consists of the homogeneous polynomials of degree $k$ in the Plücker coordinates.

Some distinguished elements of degree $\varpi_{j}$ in $\mathbb{C}\left[N^{-} \backslash G\right]$ are the generalized minors

$$
\Delta_{\varpi_{j}, w\left(\varpi_{j}\right)}, \quad(w \in W),
$$

(see $[9, \S 1.4]$ ). The image of $N_{K}$ in $B_{K}^{-} \backslash G$ under the natural projection is the open subset defined by the non-vanishing of the minors $\Delta_{\varpi_{j}, \varpi_{j}}(j \in J)$. Therefore the affine coordinate ring of $\mathbb{C}\left[N_{K}\right]$ can be identified with the subring of degree 0 homogeneous elements in the localized ring $\mathbb{C}\left[B_{K}^{-} \backslash G\right]\left[\Delta_{\varpi_{j}, \varpi_{j}}^{-1}, j \in J\right]$. Equivalently, $\mathbb{C}\left[N_{K}\right]$ can be identified with the quotient of $\mathbb{C}\left[B_{K}^{-} \backslash G\right]$ by the ideal generated by the elements $\Delta_{\varpi_{j}, \varpi_{j}}-1(j \in J)$.

Example 10.2. We continue Example 10.1. The coordinate ring of $\mathbb{C}\left[N_{K}\right]$ is isomorphic to the ring generated by the $\Delta_{i j}$ modulo the relation $\Delta_{12}=1$. This description may seem unnecessarily complicated since $N_{K}$ is just an affine space of dimension 6 and we choose a presentation with 9 generators and the Plücker relations. But these generators are better adapted to the cluster algebra structure that we shall introduce.

Let $\operatorname{pr}_{J}: \mathbb{C}\left[B_{K}^{-} \backslash G\right] \rightarrow \mathbb{C}\left[N_{K}\right]$ denote the projection obtained by modding out the ideal generated by the elements $\Delta_{\varpi_{j}, \varpi_{j}}-1(j \in J)$. If $\mathbb{C}\left[B_{K}^{-} \backslash G\right]$ is identified as explained above with a subalgebra of $\mathbb{C}[G]$, this map $\operatorname{pr}_{J}$ is nothing else than the restriction of functions from $G$ to $N_{K}$. The restriction of $\operatorname{pr}_{J}$ to each homogeneous piece $L(\lambda)\left(\lambda \in \Pi_{J}\right)$ of $\mathbb{C}\left[B_{K}^{-} \backslash G\right]$ is injective and gives an embedding of $L(\lambda)$ into $\mathbb{C}\left[N_{K}\right]$. Moreover, any $f \in \mathbb{C}\left[N_{K}\right]$ belongs to $\operatorname{pr}_{J}(L(\lambda))$ for some $\lambda \in \Pi_{J}$.

Summarizing this discussion and taking into account Theorem 8.1, we get the following description of $\mathbb{C}\left[N_{K}\right]$, convenient for our purpose.

Theorem 10.3 ([17]). Let $R_{J}$ be the subspace of $\mathbb{C}[N]$ spanned by

$$
\left\{\varphi_{M} \mid M \text { is a submodule of } Q_{\lambda} \text { for some } \lambda \in \Pi_{J}\right\} \text {. }
$$

The restriction to $R_{J}$ of the natural homomorphism $\mathbb{C}[N] \rightarrow \mathbb{C}\left[N_{K}\right]$ (given by restricting functions from $N$ to $N_{K}$ ) is an isomorphism. 


\section{The category $\operatorname{Sub} Q_{J}$}

Set $Q_{J}=\oplus_{j \in J} Q_{j}$. Let $\operatorname{Sub} Q_{J}$ denote the full subcategory of $\bmod \Lambda$ whose objects are the submodules of a direct sum of a finite number of copies of $Q_{J}$.

Example 11.1. Type $D_{4}$. We have seen in Example 3.1 the structure of the indecomposable injective $Q_{4}$. It is easy to see that $Q_{4}$ has only seven nonzero submodules

$$
S_{4}, \frac{S_{3}}{S_{4}}, \frac{S_{2}}{S_{3}}, \frac{S_{1}}{S_{4}}, \frac{S_{1} \oplus S_{2}}{S_{4}}, \frac{\frac{S_{3}}{S_{3}}}{\frac{S_{1} \oplus S_{2}}{S_{3}}}, \frac{\frac{S_{4}}{S_{4}}}{\frac{\frac{S_{3}}{S_{1} \oplus S_{2}}}{\frac{S_{3}}{S_{4}}},}
$$

which are all indecomposable. It turns out that $\operatorname{Sub} Q_{4}$ contains a unique other indecomposable object, which is a submodule of $Q_{4} \oplus Q_{4}$, and has the following socle series

$$
\frac{\frac{S_{3}}{S_{1} \oplus S_{2}}}{\frac{S_{3}}{S_{4} \oplus S_{4}}} .
$$

Every object of $\operatorname{Sub} Q_{4}$ is a sum of copies of these eight indecomposable objects.

Since $Q_{J}$ is an injective $\Lambda$-module, the category $\operatorname{Sub} Q_{J}$ has good homological properties [2]. In particular, it is closed under extensions, has enough injectives, enough projectives and almost split sequences. Moreover, the injectives coincide with the projectives (it is a Frobenius category) and its stable category is a 2Calabi-Yau triangulated category. Clearly, the algebra $R_{J}$ of Theorem 10.3 is nothing but the linear span of

$$
\left\{\varphi_{M} \mid M \in \operatorname{Sub} Q_{J}\right\}
$$

Hence we may regard $\operatorname{Sub} Q_{J}$ as a kind of "categorification" of $\mathbb{C}\left[N_{K}\right]$. We are going to make this statement more precise by studying the rigid modules in $\operatorname{Sub} Q_{J}$.

Theorem $11.2([17])$. Let $T$ be a rigid module in $\operatorname{Sub} Q_{J}$. The number of pairwise nonisomorphic indecomposable direct summands of $T$ is at most equal to $\operatorname{dim} N_{K}$.

In the case when $J=I$, that is, $\operatorname{Sub} Q_{J}=\bmod \Lambda$, this result was first obtained in $[19]$.

We shall say that a rigid module $T$ in $\operatorname{Sub} Q_{J}$ is complete if it has this maximal number of nonisomorphic summands. Note that in this case, $T$ obviously contains the $n$ indecomposable injective objects of $\operatorname{Sub} Q_{J}$.

In order to construct explicitly some complete rigid modules, we shall introduce certain functors. 


\section{The functors $\mathcal{E}_{i}$ and $\mathcal{E}_{i}^{\dagger}$}

For $i \in I$, we define an endo-functor $\mathcal{E}_{i}$ of $\bmod \Lambda$ as follows. Given an object $M \in \bmod \Lambda$ we define $\mathcal{E}_{i}(M)$ as the kernel of the surjection

$$
M \rightarrow S_{i}^{\oplus m_{i}(M)},
$$

where $m_{i}(M)$ denotes the multiplicity of $S_{i}$ in the top of $M$. If $f: M \rightarrow N$ is a homomorphism, $f\left(\mathcal{E}_{i}(M)\right)$ is contained in $\mathcal{E}_{i}(N)$, and we define

$$
\mathcal{E}_{i}(f): \mathcal{E}_{i}(M) \rightarrow \mathcal{E}_{i}(N)
$$

as the restriction of $f$ to $\mathcal{E}_{i}(M)$. Clearly, $\mathcal{E}_{i}$ is an additive functor. It acts on a module $M$ by removing the $S_{i}$-isotypical part of its top. Similarly, one can define a functor $\mathcal{E}_{i}^{\dagger}$ acting on $M$ by removing the $S_{i}$-isotypical part of its socle.

Proposition 12.1 ([17]). The functors $\mathcal{E}_{i}, \mathcal{E}_{i}^{\dagger}(i \in I)$ satisfy the following relations:

(i) $\mathcal{E}_{i} \mathcal{E}_{i}=\mathcal{E}_{i}, \quad \mathcal{E}_{i}^{\dagger} \mathcal{E}_{i}^{\dagger}=\mathcal{E}_{i}^{\dagger}$.

(ii) $\mathcal{E}_{i} \mathcal{E}_{j}=\mathcal{E}_{j} \mathcal{E}_{i}, \quad \mathcal{E}_{i}^{\dagger} \mathcal{E}_{j}^{\dagger}=\mathcal{E}_{j}^{\dagger} \mathcal{E}_{i}^{\dagger}$, if $i$ and $j$ are not connected by an edge in $\Delta$.

(iii) $\mathcal{E}_{i} \mathcal{E}_{j} \mathcal{E}_{i}=\mathcal{E}_{j} \mathcal{E}_{i} \mathcal{E}_{j}, \quad \mathcal{E}_{i}^{\dagger} \mathcal{E}_{j}^{\dagger} \mathcal{E}_{i}^{\dagger}=\mathcal{E}_{j}^{\dagger} \mathcal{E}_{i}^{\dagger} \mathcal{E}_{j}^{\dagger}$, if $i$ and $j$ are connected by an edge.

Relations (ii) and (iii) are the braid relations for $\Delta$. It follows that for any element $w$ of the Weyl group $W$ of $G$, we have well-defined functors

$$
\mathcal{E}_{w}:=\mathcal{E}_{i_{1}} \cdots \mathcal{E}_{i_{k}}, \quad \mathcal{E}_{w}^{\dagger}:=\mathcal{E}_{i_{1}}^{\dagger} \cdots \mathcal{E}_{i_{k}}^{\dagger},
$$

where $w=s_{i_{1}} \cdots s_{i_{k}}$ is an arbitrary reduced decomposition of $w$.

Consider now the parabolic subgroup $W_{K}$ of $W$ generated by the $s_{k}(k \in K)$. This is a finite Coxeter group. Let $w_{0}^{K}$ denote its longest element. One can check that $\mathcal{E}_{w_{0}^{K}}^{\dagger}(M) \in \operatorname{Sub} Q_{J}$ for every $M \in \bmod \Lambda$, and that $\mathcal{E}_{w_{0}^{K}}^{\dagger}(M)=M$ if $M \in \operatorname{Sub} Q_{J}$. In other words, the subcategory $\operatorname{Sub} Q_{J}$ can be described as the image of $\bmod \Lambda$ under the endo-functor $\mathcal{E}_{w_{0}^{K}}^{\dagger}[17]$.

\section{Construction of complete rigid modules}

The relevance of these functors for constructing rigid modules comes from the following property.

Proposition 13.1 ([17]). The functors $\mathcal{E}_{w}$ and $\mathcal{E}_{w}^{\dagger}$ preserve rigid modules, i.e. if $M$ is rigid then $\mathcal{E}_{w}(M)$ and $\mathcal{E}_{w}^{\dagger}(M)$ are also rigid.

Let $w_{0}$ be the longest element of $W$, and let $w_{0}=s_{i_{1}} \cdots s_{i_{r}}$ be a reduced decomposition such that the first $r_{K}$ factors form a reduced decomposition of $w_{0}^{K}$. Set

$$
u_{\leq p}=s_{i_{1}} \cdots s_{i_{p}}, \quad M_{p}=\mathcal{E}_{u_{\leq p}}^{\dagger}\left(Q_{i_{p}}\right), \quad(p=1, \ldots, r) .
$$


For $k \in K$, let $q_{k}=\max \left\{q \leq r_{K} \mid i_{q}=k\right\}$. Finally, define

$$
T=M_{r_{K}+1} \oplus M_{r_{K}+2} \oplus \cdots \oplus M_{r} \oplus\left(\oplus_{k \in K} M_{q_{k}}\right) \oplus Q_{J} .
$$

Theorem $13.2([17]) . T$ is a complete rigid module in $\operatorname{Sub} Q_{J}$.

Note that by construction the modules $M_{p}$ with $p>r_{K}$ are in the image of the functor $\mathcal{E}_{w_{0}^{K}}^{\dagger}$, hence in $\operatorname{Sub} Q_{J}$. Note also that $M_{q_{k}}=\mathcal{E}_{w_{0}^{K}}^{\dagger}\left(Q_{k}\right)$ for $k \in K$. The modules $M_{q_{k}}$ together with the summands of $Q_{J}$ are the indecomposable injectives of Sub $Q_{J}$. Finally, if $t_{l}=\max \left\{t \leq r \mid i_{t}=l\right\}$, then $M_{t_{l}}=\mathcal{E}_{w_{0}}^{\dagger}\left(Q_{l}\right)=0$ for every $l \in I$. It follows that $T$ has in fact $r-r_{K}+|K|+|J|-|I|=r-r_{K}=\operatorname{dim} N_{K}$ indecomposable summands, in agreement with Theorem 11.2.

Example 13.3. Type $D_{4}$. We take again $K=\{1,2,3\}, J=\{4\}$ (remember that the central node of $\Delta$ is labelled by 3 ). Here $r_{K}=6$. We choose the reduced decomposition

$$
w_{0}=s_{1} s_{3} s_{1} s_{2} s_{3} s_{1} s_{4} s_{3} s_{1} s_{2} s_{3} s_{4}
$$

We then have

$$
\begin{gathered}
M_{s_{2}}=M_{4}=\frac{\frac{S_{2}}{S_{3}}}{S_{4}}, \quad M_{s_{3}}=M_{5}=\frac{\frac{S_{3}}{\frac{S_{1} \oplus S_{2}}{S_{3}}}, \quad M_{s_{1}}=M_{6}=\frac{S_{1}}{S_{3} \oplus S_{4}}}{S_{4}}, \\
M_{7}=S_{4}, \quad M_{8}=\frac{S_{3}}{S_{4}}, \quad M_{9}=M_{10}=M_{11}=M_{12}=0 .
\end{gathered}
$$

The module $T=M_{4} \oplus M_{5} \oplus M_{6} \oplus M_{7} \oplus M_{8} \oplus Q_{4}$ is complete rigid in $\operatorname{Sub} Q_{4}$.

\section{Cluster algebras of geometric type}

Our next aim will be to associate to the category $\operatorname{Sub} Q_{J}$ certain cluster algebras of geometric type. We refer the reader to $[10,11,3]$ for a detailed exposition of their properties and of the motivating examples of coordinate rings of double Bruhat cells. Here we shall merely recall their definition.

Let $d$ and $n$ be integers with $d \geq n \geq 0$. If $B=\left(b_{i j}\right)$ is any $d \times(d-n)$-matrix with integer entries, then the principal part of $B$ is obtained by deleting from $B$ the last $n$ rows. Given some $k \in[1, d-n]$ define a new $d \times(d-n)$-matrix $\mu_{k}(B)=\left(b_{i j}^{\prime}\right)$ by

$$
b_{i j}^{\prime}= \begin{cases}-b_{i j} & \text { if } i=k \text { or } j=k, \\ b_{i j}+\frac{\left|b_{i k}\right| b_{k j}+b_{i k}\left|b_{k j}\right|}{2} & \text { otherwise, }\end{cases}
$$

where $i \in[1, d]$ and $j \in[1, d-n]$. One calls $\mu_{k}(B)$ the mutation of $B$ in direction $k$. If $B$ is an integer matrix whose principal part is skew-symmetric, then it is easy to check that $\mu_{k}(B)$ is also an integer matrix with skew-symmetric principal part. 
In this case, Fomin and Zelevinsky define a cluster algebra $\mathcal{A}(B)$ as follows. Let $\mathcal{F}=\mathbb{C}\left(y_{1}, \ldots, y_{d}\right)$ be the field of rational functions in $d$ commuting variables $\mathbf{y}=\left(y_{1}, \ldots, y_{d}\right)$. One calls $(\mathbf{y}, B)$ the initial seed of $\mathcal{A}(B)$. For $1 \leq k \leq d-n$ define

$$
y_{k}^{*}=\frac{\prod_{b_{i k}>0} y_{i}^{b_{i k}}+\prod_{b_{i k}<0} y_{i}^{-b_{i k}}}{y_{k}} .
$$

Let $\mu_{k}(\mathbf{y})$ denote the $d$-tuple obtained from $\mathbf{y}$ by replacing $y_{k}$ by $y_{k}^{*}$. The pair $\left(\mu_{k}(\mathbf{y}), \mu_{k}(B)\right)$ is the mutation of the seed $(\mathbf{y}, B)$ in direction $k$.

Now one can iterate this process and mutate again each seed $\left(\mu_{k}(\mathbf{y}), \mu_{k}(B)\right)$ in $d-n$ directions. The collection of all seeds obtained from the initial seed $(\mathbf{y}, B)$ via a finite sequence of mutations is called the mutation class of $(\mathbf{y}, B)$. Each seed in this class consists of a $d$-tuple of algebraically independent elements of $\mathcal{F}$ called a cluster and of a matrix called the exchange matrix. The elements of a cluster are its cluster variables. One does not mutate the last $n$ elements of a cluster. They are called coefficients and belong to every cluster. The cluster algebra $\mathcal{A}(B)$ is by definition the subalgebra of $\mathcal{F}$ generated by the set of all the cluster variables appearing in a seed of the mutation class of $(\mathbf{y}, B)$. The subring of $\mathcal{A}(B)$ generated by the coefficients is called the coefficient ring. The integer $d-n$ is called the rank of $\mathcal{A}(B)$. A monomial in the cluster variables is called a cluster monomial if all its variables belong to a single cluster.

Example 14.1. Take $d=7$ and $n=5$. Let

$$
B=\left[\begin{array}{cc}
0 & -1 \\
1 & 0 \\
-1 & 0 \\
1 & 0 \\
-1 & 1 \\
0 & -1 \\
0 & 1
\end{array}\right]
$$

Then the mutation in direction 1 reads

$$
\mu_{1}(B)=\left[\begin{array}{cc}
0 & 1 \\
-1 & 0 \\
1 & -1 \\
-1 & 0 \\
1 & 0 \\
0 & -1 \\
0 & 1
\end{array}\right], \quad y_{1}^{*}=\frac{y_{2} y_{4}+y_{3} y_{5}}{y_{1}} .
$$

In this simple example, it turns out that $\mathcal{A}(B)$ has only a finite number of cluster variables. In fact $\mathcal{A}(B)$ is isomorphic to the homogeneous coordinate ring of the Grassmannian $\operatorname{Gr}(2,5)$ of 2-planes of $\mathbb{C}^{5}[11, \S 12]$. The explicit isomorphism maps the cluster variables $y_{1}, \ldots, y_{7}$ to the following Plücker coordinates:

$y_{1} \mapsto[1,3], y_{2} \mapsto[1,4], y_{3} \mapsto[1,2], y_{4} \mapsto[2,3], y_{5} \mapsto[3,4], y_{6} \mapsto[4,5], y_{7} \mapsto[1,5]$. 
The other cluster variables obtained by mutation from this initial seed are the remaining Plücker coordinates $[2,4],[2,5],[3,5]$.

\section{Mutation of complete rigid modules}

We shall now introduce an operation of mutation for complete rigid modules in $\operatorname{Sub} Q_{J}$, inspired by the cluster algebra mutation of Fomin and Zelevinsky.

Let $T=T_{1} \oplus \cdots \oplus T_{d}$ be an arbitrary basic complete rigid module in Sub $Q_{J}$. Thus the $T_{i}$ 's are indecomposable and pairwise non isomorphic, and

$$
d=r-r_{K}=\ell\left(w_{0}\right)-\ell\left(w_{0}^{K}\right)=\ell\left(w_{0}^{K} w_{0}\right) .
$$

Assume that the injective summands of $T$ are the last $n$ ones, namely $T_{d-n+1}, \ldots, T_{d}$. Relying on the results of [14] we show in [17] the following

Theorem 15.1. Let $k \leq d-n$. There exists a unique indecomposable module $T_{k}^{*} \neq T_{k}$ in $\operatorname{Sub} Q_{J}$ such that $\left(T / T_{k}\right) \oplus T_{k}^{*}$ is a basic complete rigid module in $\operatorname{Sub} Q_{J}$. Moreover, $\operatorname{dim} \operatorname{Ext}_{\Lambda}^{1}\left(T_{k}, T_{k}^{*}\right)=1$ and if

$$
0 \rightarrow T_{k} \stackrel{g}{\rightarrow} X_{k} \stackrel{f}{\rightarrow} T_{k}^{*} \rightarrow 0, \quad 0 \rightarrow T_{k}^{*} \stackrel{i}{\rightarrow} Y_{k} \stackrel{h}{\rightarrow} T_{k} \rightarrow 0
$$

are the unique non-split short exact sequences between $T_{k}$ and $T_{k}^{*}$, then $f, g, h, i$ are minimal $\operatorname{add}\left(T / T_{k}\right)$-approximations, and $X_{k}$ and $Y_{k}$ have no isomorphic indecomposable summands.

In this situation, we say that $\left(T / T_{k}\right) \oplus T_{k}^{*}$ is the mutation of $T$ in the direction of $T_{k}$, and we write $\mu_{k}(T)=\left(T / T_{k}\right) \oplus T_{k}^{*}$. Since $X_{k}$ and $Y_{k}$ belong to $\operatorname{add}(T)$, we can describe this mutation by means of the multiplicities of each indecomposable summand of $T$ in $X_{k}$ and $Y_{k}$. This leads to associate to $T$ a matrix of integers called its exchange matrix encoding the mutations of $T$ in all possible $d-n$ directions.

More precisely, define $b_{i k}=\left[X_{k}: T_{i}\right]$ if $X_{k}$ has summands isomorphic to $T_{i}$, $b_{i k}=-\left[Y_{k}: T_{i}\right]$ if $Y_{k}$ has summands isomorphic to $T_{i}$, and $b_{i k}=0$ otherwise. Note that these conditions are disjoint because $X_{k}$ and $Y_{k}$ have no isomorphic direct summands. The $d \times(d-n)$ matrix $B(T)=\left[b_{i k}\right]$ is called the exchange matrix of $T$. We can now state:

Theorem $15.2([14,17])$. Let $T=T_{1} \oplus \cdots \oplus T_{d}$ be a complete rigid module in Sub $Q_{J}$ as above, and let $k \leq d-n$. Then

$$
B\left(\mu_{k}(T)\right)=\mu_{k}(B(T)),
$$

where on the right-hand side $\mu_{k}$ stands for the Fomin-Zelevinsky matrix mutation.

In other words, our mutation of complete rigid modules induces at the level of exchange matrices the Fomin-Zelevinsky matrix mutation. 


\section{Cluster algebra structure on $\mathbb{C}\left[N_{K}\right]$}

Let $T$ be one of the complete rigid modules of $\S 13$. Consider the mutation class $\mathcal{R}$ of $T$, that is, the set of all complete rigid modules of $\operatorname{Sub} Q_{J}$ which can be obtained from $T$ by a finite sequence of mutations. One can show that $\mathcal{R}$ contains all the rigid modules of $\S 13$ corresponding to all possible choices of a reduced decomposition of $w_{0}^{K} w_{0}$, hence $\mathcal{R}$ does not depend on the choice of $T$.

We can now project $\mathcal{R}$ on $R_{J} \cong \mathbb{C}\left[N_{K}\right]$ using the map $M \mapsto \varphi_{M}$. More precisely, for $U=U_{1} \oplus \cdots \oplus U_{d} \in \mathcal{R}$, let $x(U)=\left(\varphi_{U_{1}}, \ldots, \varphi_{U_{d}}\right)$, (where again $\left.d=r-r_{K}\right)$. The next result follows from Theorem 15.1 and Theorem 6.1.

Theorem 16.1. (i) $\{x(U) \mid U \in \mathcal{R}\}$ is the set of clusters of a cluster algebra $\mathcal{A}_{J} \subseteq R_{J} \cong \mathbb{C}\left[N_{K}\right]$ of rank $d-n$.

(ii) The coefficient ring of $\mathcal{A}_{J}$ is the ring of polynomials in the $n$ variables $\varphi_{L_{i}}(i \in I)$, where the $L_{i}$ are the indecomposable injective objects in Sub $Q_{J}$.

(iii) All the cluster monomials belong to the dual semicanonical basis of $\mathbb{C}[N]$, and are thus linearly independent.

The rigid modules $T$ of $\S 13$ project to initial seeds of the cluster algebra $\mathcal{A}_{J}$ that we are going to describe.

For $i \in I$ and $u, v \in W$, let $\Delta_{u\left(\varpi_{i}\right), v\left(\varpi_{i}\right)}$ denote the generalized minor introduced by Fomin and Zelevinsky [9, §1.4]. This is a regular function on $G$. We shall mainly work with the restriction of this function to $N$, that we shall denote by $D_{u\left(\varpi_{i}\right), v\left(\varpi_{i}\right)}$. It is easy to see that $D_{u\left(\varpi_{i}\right), v\left(\varpi_{i}\right)}=0$ unless $u\left(\varpi_{i}\right)$ is less or equal to $v\left(\varpi_{i}\right)$ in the Bruhat order, and that $D_{u\left(\varpi_{i}\right), u\left(\varpi_{i}\right)}=1$ for every $i \in I$ and $u \in W$. It is also well known that $D_{\varpi_{i}, w_{0}\left(\varpi_{i}\right)}$ is a lowest weight vector of $L\left(\varpi_{i}\right)$ in its realization as a subspace of $\mathbb{C}[N]$ explained in $\S 8$. Therefore, using Theorem 8.1 , we get

$$
\varphi_{Q_{i}}=D_{\varpi_{i}, w_{0}\left(\varpi_{i}\right)}, \quad(i \in I) .
$$

More generally, it follows from [15, Lemma 5.4] that for $u, v \in W$ we have

$$
\varphi_{\mathcal{E}_{u}^{\dagger} \mathcal{E}_{v} Q_{i}}=D_{u\left(\varpi_{i}\right), v w_{0}\left(\varpi_{i}\right)}
$$

Thus the elements of $\mathbb{C}[N]$ attached to the summands $M_{p}$ of the complete rigid module $T$ of Theorem 13.2 are given by

$$
\begin{aligned}
\varphi_{M_{p}} & =D_{\varpi_{i_{p}}, u_{\leq p} w_{0}\left(\varpi_{i_{p}}\right),} & & \left(p \in\left\{r_{K}+1, \ldots, r\right\} \cup\left\{s_{k} \mid k \in K\right\}\right), \\
\varphi_{Q_{j}} & =D_{\varpi_{j}, w_{0}\left(\varpi_{j}\right),} & & (j \in J) .
\end{aligned}
$$

Moreover the matrix $B(T)$ can also be described explicitly by means of a graph similar to those arising in the Chamber Ansatz of Fomin and Zelevinsky (see [17, $\S 9.3])$. 
Example 16.2. We continue Example 13.3. We have

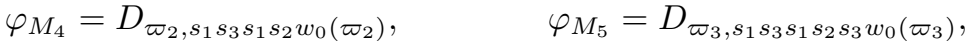

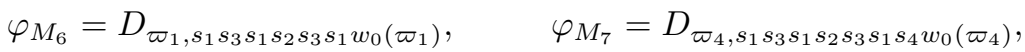

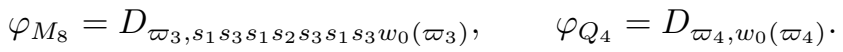

It turns out that in the matrix realization of $N$ given in Example 4.2 the generalized minors above can be expressed as ordinary minors of the unitriangular $8 \times 8$ matrix $x \in N$. Indeed, denoting the matrix entries of $x$ by $n_{i j}(x)$ one can check that

$$
\begin{array}{lll}
\varphi_{M_{4}}=n_{14}, & \varphi_{M_{5}}=\left|\begin{array}{cc}
n_{17} & n_{18} \\
1 & n_{78}
\end{array}\right|, & \varphi_{M_{6}}=n_{15}, \\
\varphi_{M_{7}}=n_{12}, & \varphi_{M_{8}}=n_{13}, & \varphi_{Q_{4}}=n_{18} .
\end{array}
$$

The cluster variables of this seed are $\varphi_{M_{7}}$ and $\varphi_{M_{8}}$. The exchange relations come from the following exact sequences

$$
\begin{gathered}
0 \rightarrow M_{7} \rightarrow M_{5} \rightarrow M_{7}^{*} \rightarrow 0, \quad 0 \rightarrow M_{7}^{*} \rightarrow Q_{4} \rightarrow M_{7} \rightarrow 0, \\
0 \rightarrow M_{8} \rightarrow M_{4} \oplus M_{6} \rightarrow M_{8}^{*} \rightarrow 0, \quad 0 \rightarrow M_{8}^{*} \rightarrow M_{5} \rightarrow M_{8} \rightarrow 0,
\end{gathered}
$$

where

$$
M_{7}^{*}=\frac{\frac{S_{3}}{S_{1} \oplus S_{2}}}{\frac{S_{3}}{S_{4}}} \quad M_{8}^{*}=\frac{\frac{S_{1} \oplus S_{2}}{S_{3}}}{S_{4}} .
$$

The exchange matrix is therefore

$$
B(T)=\left[\begin{array}{cc}
0 & 0 \\
0 & 0 \\
0 & -1 \\
-1 & 1 \\
0 & -1 \\
1 & 0
\end{array}\right]
$$

where the rows are labelled by $\left(M_{7}, M_{8}, M_{4}, M_{5}, M_{6}, Q_{4}\right)$ and the columns by $\left(M_{7}, M_{8}\right)$.

A priori, we only have an inclusion of our cluster algebra $\mathcal{A}_{J}$ in $R_{J} \cong \mathbb{C}\left[N_{K}\right]$, but we believe that

Conjecture 16.3. We have $\mathcal{A}_{J}=R_{J}$.

The conjecture is proved for $G$ of type $A_{n}$ and of type $D_{4}$. It is also proved for $J=\{n\}$ in type $D_{n}$, and for $J=\{1\}$ in type $D_{5}$ (see [17]). Moreover it follows from [18] that it is also true whenever $w_{0}^{K} w_{0}$ has a reduced expression adapted to an orientation of the Dynkin diagram. 


\section{Cluster algebra structure on $\mathbb{C}\left[B_{K}^{-} \backslash G\right]$}

Let us start by some simple remarks. Consider the affine space $\mathbb{C}^{r}$ and the projective space $\mathbb{P}\left(\mathbb{C}^{r+1}\right)$. The coordinate ring of $\mathbb{C}^{r}$ is $R=\mathbb{C}\left[x_{1}, \ldots, x_{r}\right]$, and the homogeneous coordinate ring of $\mathbb{P}\left(\mathbb{C}^{r+1}\right)$ is $S=\mathbb{C}\left[x_{1}, \ldots, x_{r+1}\right]$. Moreover $\mathbb{C}^{r}$ can be regarded as the open subset of $\mathbb{P}\left(\mathbb{C}^{r+1}\right)$ given by the non-vanishing of $x_{r+1}$. Given a hypersurface $\Sigma \subset \mathbb{C}^{r}$ of equation $f\left(x_{1}, \ldots, x_{r}\right)=0$ for some $f \in R$, its completion $\widehat{\Sigma} \subset \mathbb{P}\left(\mathbb{C}^{r+1}\right)$ is described by the equation $\widehat{f}\left(x_{1}, \ldots, x_{r+1}\right)=0$, where $\widehat{f}$ is the homogeneous element of $S$ obtained by multiplying each monomial of $f$ by an appropriate power of $x_{r+1}$.

Similarly, consider the open embedding $N_{K} \subset B_{K}^{-} \backslash G$ given by restricting the natural projection $G \rightarrow B_{K}^{-} \backslash G$ to $N_{K}$. By this embedding, $N_{K}$ is identified with the subset of $B_{K}^{-} \backslash G$ given by the simultaneous non-vanishing of the generalized minors $\Delta_{\varpi_{j}, \varpi_{j}}(j \in J)$. To an element $f \in \mathbb{C}\left[N_{K}\right]$ we can associate a $\Pi_{J}$-homogeneous element $\tilde{f} \in \mathbb{C}\left[B_{K}^{-} \backslash G\right]$ by multiplying each monomial in $f$ by an appropriate monomial in the $\Delta_{\varpi_{j}, \varpi_{j}}$ 's. More precisely, using the notation of $\S 10, \tilde{f}$ is the homogeneous element of $\mathbb{C}\left[B_{K}^{-} \backslash G\right]$ with smallest degree such that $\operatorname{pr}_{J}(\widetilde{f})=f$.

With this notation, we can now state the following result of [17].

Theorem 17.1. (i) $\{\widetilde{x(U)} \mid U \in \mathcal{R}\}$ is the set of clusters of a cluster algebra $\widetilde{\mathcal{A}}_{J} \subseteq \mathbb{C}\left[B_{K}^{-} \backslash G\right]$ of rank $d-n$.

(ii) The coefficient ring of $\widetilde{\mathcal{A}}_{J}$ is the ring of polynomials in the $n+|J|$ variables $\widetilde{\varphi_{L_{i}}}(i \in I)$ and $\Delta_{\varpi_{j}, \varpi_{j}}(j \in J)$.

(iii) The exchange matrix $\widetilde{B}$ attached to $\widetilde{x(U)}$ is obtained from the exchange matrix $B$ of $x(U)$ by adding $|J|$ new rows (in the non-principal part) labelled by $j \in J$, where the entry in column $k$ and row $j$ is equal to

$$
b_{j k}=\operatorname{dim} \operatorname{Hom}_{\Lambda}\left(S_{j}, X_{k}\right)-\operatorname{dim} \operatorname{Hom}_{\Lambda}\left(S_{j}, Y_{k}\right) .
$$

Here, if $U_{k}$ denotes the $k$ th summand of $U, U_{k}^{*}$ its mutation, then $X_{k}, Y_{k}$ are the middle terms of the non-split short exact sequences

$$
0 \rightarrow U_{k} \rightarrow X_{k} \rightarrow U_{k}^{*} \rightarrow 0, \quad 0 \rightarrow U_{k}^{*} \rightarrow Y_{k} \rightarrow U_{k} \rightarrow 0 .
$$

Example 17.2. We continue Example 13.3 and Example 16.2. So we are in type $D_{4}$ with $K=\{1,2,3\}$ and $J=\{4\}$. The cluster $\widetilde{x(T)}$ consists of the 7 functions

$$
\widetilde{\varphi_{M_{4}}}, \widetilde{\varphi_{M_{5}}}, \widetilde{\varphi_{M_{6}}}, \widetilde{\varphi_{M_{7}}}, \widetilde{\varphi_{M_{8}}}, \widetilde{\varphi_{Q_{4}}}, \Delta_{\varpi_{4}, \varpi_{4}} \text {. }
$$

The exchange matrix $\widetilde{B(T)}$ is obtained from the matrix $B(T)$ of Example 16.2 by adding a new row labelled by the extra coefficient $\Delta_{\varpi_{4}, \varpi_{4}}$. Since

$$
\operatorname{dim} \operatorname{Hom}_{\Lambda}\left(S_{4}, M_{5}\right)-\operatorname{dim} \operatorname{Hom}_{\Lambda}\left(S_{4}, Q_{4}\right)=2-1=1,
$$




$$
\operatorname{dim} \operatorname{Hom}_{\Lambda}\left(S_{4}, M_{4} \oplus M_{6}\right)-\operatorname{dim} \operatorname{Hom}_{\Lambda}\left(S_{4}, M_{5}\right)=2-2=0,
$$

this new row is equal to $[1,0]$, thus

$$
\widetilde{B(T)}=\left[\begin{array}{cc}
0 & 0 \\
0 & 0 \\
0 & -1 \\
-1 & 1 \\
0 & -1 \\
1 & 0 \\
1 & 0
\end{array}\right] .
$$

Note that in this example, the variety $B_{K}^{-} \backslash G$ is isomorphic to a smooth quadric in $\mathbb{P}\left(\mathbb{C}^{8}\right)$. Its homogeneous coordinate ring $\mathbb{C}\left[B_{K}^{-} \backslash G\right]$ coincides with the affine coordinate ring of the isotropic cone in $\mathbb{C}^{8}$ of the corresponding non-degenerate quadratic form. Thus we recover an example of $\S 2$. The precise identification is via the following formulas (see Exercise 5.7):

$$
\begin{gathered}
y_{1}=\Delta_{\varpi_{4}, \varpi_{4}}, y_{2}=\widetilde{\varphi_{M_{7}}}, y_{3}=\widetilde{\varphi_{M_{8}}}, y_{4}=\widetilde{\varphi_{M_{4}}}, y_{5}=\widetilde{\varphi_{M_{6}}}, \\
y_{6}=\widetilde{\varphi_{M_{8}^{*}}}, y_{7}=\widetilde{\varphi_{M_{7}^{*}}}, y_{8}=\widetilde{\varphi_{Q_{4}}}, p=\widetilde{\varphi_{M_{5}}} .
\end{gathered}
$$

Note that since $\mathbb{C}\left[B_{K}^{-} \backslash G\right]$ is generated by the $y_{i}(1 \leq i \leq 8)$, which are cluster variables or generators of the coefficient ring of $\widetilde{\mathcal{A}}_{J}$, we have in this case that $\widetilde{\mathcal{A}}_{J}=\mathbb{C}\left[B_{K}^{-} \backslash G\right]$.

When $J=\{j\}$ and $G$ is of type $A, B_{K}^{-} \backslash G$ is a Grassmannian and the cluster algebra $\widetilde{\mathcal{A}}_{J}$ coincides with the one defined by Scott in [34].

When $K=\emptyset, \mathbb{C}\left[B_{K}^{-} \backslash G\right]=\mathbb{C}\left[N^{-} \backslash G\right]$ and $J=I$ the cluster algebra $\widetilde{\mathcal{A}}_{J}$ is essentially the same as the one attached by Berenstein, Fomin and Zelevinsky to the big cell of the base affine space $N^{-} \backslash G$ in $[3, \S 2.6]$. More precisely, both cluster algebras have identical seeds, but Berenstein, Fomin and Zelevinsky consider an upper cluster algebra, and they assume that the coefficients

$$
\widetilde{\varphi_{L_{i}}}=\widetilde{\varphi_{Q_{i}}}=\Delta_{\varpi_{i}, w_{0}\left(\varpi_{i}\right)}, \quad \Delta_{\varpi_{i}, \varpi_{i}}, \quad(i \in I),
$$

are invertible, i.e. the ring of coefficients consists of Laurent polynomials.

Let $\Sigma_{J}$ be the multiplicative submonoid of $\widetilde{\mathcal{A}}_{J}$ generated by the set

$$
\left\{\Delta_{\varpi_{j}, \varpi_{j}} \mid j \in J \text { and } \varpi_{j} \text { is not a minuscule weight }\right\} .
$$

Conjecture 17.3. The localizations of $\widetilde{\mathcal{A}}_{J}$ and $\mathbb{C}\left[B_{K}^{-} \backslash G\right]$ with respect to $\Sigma_{J}$ are equal.

Note that if $J$ is such that all the weights $\varpi_{j}(j \in J)$ are minuscule, then $\Sigma_{J}$ is trivial and the conjecture states that the algebras $\widetilde{\mathcal{A}}_{J}$ and $\mathbb{C}\left[B_{K}^{-} \backslash G\right]$ coincide without localization. This is in particular the case for every $J$ in type $A_{n}$.

The conjecture is proved for $G$ of type $A_{n}$ and of type $D_{4}$. It is also proved for $J=\{n\}$ in type $D_{n}$, and for $J=\{1\}$ in type $D_{5}$ (see [17]). Note also that Conjecture 17.3 implies Conjecture 16.3. 


\begin{tabular}{|c|c|c|}
\hline Type of $G$ & $J$ & Type of $\mathcal{A}_{J}$ \\
\hline$A_{n}(n \geq 2)$ & $\{1\}$ & - \\
$A_{n}(n \geq 2)$ & $\{2\}$ & $A_{n-2}$ \\
$A_{n}(n \geq 2)$ & $\{1,2\}$ & $A_{n-1}$ \\
$A_{n}(n \geq 2)$ & $\{1, n\}$ & $\left(A_{1}\right)^{n-1}$ \\
$A_{n}(n \geq 3)$ & $\{1, n-1\}$ & $A_{2 n-4}$ \\
$A_{n}(n \geq 3)$ & $\{1,2, n\}$ & $A_{2 n-3}$ \\
\hline$A_{4}$ & $\{2,3\}$ & $D_{4}$ \\
$A_{4}$ & $\{1,2,3\}$ & $D_{5}$ \\
$A_{4}$ & $\{1,2,3,4\}$ & $D_{6}$ \\
\hline$A_{5}$ & $\{3\}$ & $D_{4}$ \\
$A_{5}$ & $\{1,3\}$ & $E_{6}$ \\
$A_{5}$ & $\{2,3\}$ & $E_{6}$ \\
$A_{5}$ & $\{1,2,3\}$ & $E_{7}$ \\
\hline$A_{6}$ & $\{3\}$ & $E_{6}$ \\
$A_{6}$ & $\{2,3\}$ & $E_{8}$ \\
\hline$A_{7}$ & $\{3\}$ & $E_{8}$ \\
\hline$D_{n}(n \geq 4)$ & $\{n\}$ & $\left(A_{1}\right)^{n-2}$ \\
\hline$D_{4}$ & $\{1,2\}$ & $A_{5}$ \\
\hline$D_{5}$ & $\{1\}$ & $A_{5}$ \\
\hline
\end{tabular}

Table 1. Algebras $\mathcal{A}_{J}$ of finite cluster type.

\section{Finite type classification}

Recall that a cluster algebra is said to be of finite type if it has finitely many cluster variables, or equivalently, finitely many clusters. Fomin and Zelevinsky have classified the cluster algebras of finite type [11], attaching to them a finite root system called their cluster type.

Note that the clusters of $\mathcal{A}_{J}$ and $\widetilde{\mathcal{A}}_{J}$ are in natural one-to-one correspondence, and that the principal parts of the exchange matrices of two corresponding clusters are the same. This shows that $\mathcal{A}_{J}$ and $\mathcal{A}_{J}$ have the same cluster type, finite or infinite.

Using the explicit initial seed described in $\S 16$ it is possible to give a complete list of the algebras $\mathcal{A}_{J}$ which have a finite cluster type [17]. The results are summarized in Table 1. Here, we label the vertices of the Dynkin diagram of type $D_{n}$ as follows:

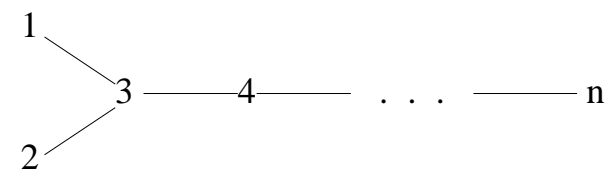

We have only listed one representative of each orbit under a diagram automorphism. For example, in type $A_{n}$ we have an order 2 diagram automorphism map- 
ping $J=\{1,2\}$ to $J^{\prime}=\{n-1, n\}$. Clearly, $\mathcal{A}_{J^{\prime}}$ has the same cluster type as $J$, namely $A_{n-1}$.

The classification when $J=I$ (that is, in the case of $\mathbb{C}[N]$ or $\mathbb{C}\left[B^{-} \backslash G\right]$ ) was given by Berenstein, Fomin and Zelevinsky [3]. The only finite type cases are $A_{n}(n \leq 4)$. The classification when $J=\{j\}$ is a singleton and $G$ is of type $A_{n}$ (the Grassmannian $\operatorname{Gr}(j, n+1)$ ) was given by Scott [34]. When $J=\{1\}$ (the projective space $\left.\mathbb{P}\left(\mathbb{C}^{n+1}\right)\right)$, the cluster algebra is trivial, since every indecomposable object of $\operatorname{Sub} Q_{1}$ is a relative projective.

Note that if $\operatorname{Sub} Q_{J}$ has finitely many isomorphism classes of indecomposable objects then by construction $\mathcal{A}_{J}$ has finite cluster type. The converse is also true although not so obvious. Indeed, if $\mathcal{A}_{J}$ has finite cluster type, then by using the classification theorem of Fomin and Zelevinsky [11] there exists a complete rigid object of $\operatorname{Sub} Q_{J}$ whose endomorphism ring has a Gabriel quiver with stable part of Dynkin type. Using a theorem of Keller and Reiten [23], it follows that the stable category $\underline{\operatorname{Sub}} Q_{J}$ is triangle equivalent to a cluster category of Dynkin type, hence $\operatorname{Sub} Q_{J}$ has finitely many indecomposable objects. Therefore the above classification is also the classification of all subcategories $\operatorname{Sub} Q_{J}$ with finitely many indecomposable objects.

\section{Canonical bases, total positivity and open pro- blems}

Since we started this survey with a discussion of total positivity and canonical bases, it is natural to ask if the previous constructions give a better understanding of these topics.

So let $\mathcal{B}$ and $\mathcal{S}$ denote respectively the dual canonical and dual semicanonical bases of $\mathbb{C}[N]$. We have seen (see $\S 7$ ) that for every rigid $\Lambda$-module $M$, the function $\varphi_{M}$ belongs to $\mathcal{S}$.

Conjecture 19.1. For every rigid $\Lambda$-module $M$, the function $\varphi_{M}$ belongs to $\mathcal{B}$.

The conjecture holds in type $A_{n}(n \leq 4)$ [12], that is, when $\Lambda$ has finite representation type. In this case one even has $\mathcal{B}=\mathcal{S}$.

As explained in $\S 8$, each finite-dimensional irreducible $G$-module $L(\lambda)$ has a canonical embedding in $\mathbb{C}[N]$. It is known that the subsets

$$
\mathcal{B}(\lambda)=\mathcal{B} \cap L(\lambda), \quad \mathcal{S}(\lambda)=\mathcal{S} \cap L(\lambda),
$$

are bases of $L(\lambda)$. Using the multiplicity-free decomposition (see $\S 10$ )

$$
\mathbb{C}\left[B_{K}^{-} \backslash G\right]=\bigoplus_{\lambda \in \Pi_{J}} L(\lambda)
$$

we therefore obtain a dual canonical and a dual semicanonical basis of $\mathbb{C}\left[B_{K}^{-} \backslash G\right]$ :

$$
\mathcal{B}_{J}=\cup_{\lambda \in \Pi_{J}} \mathcal{B}(\lambda), \quad \mathcal{S}_{J}=\cup_{\lambda \in \Pi_{J}} \mathcal{S}(\lambda) .
$$


It follows from our construction that all the cluster monomials of the cluster algebra $\widetilde{\mathcal{A}}_{J} \subset \mathbb{C}\left[B_{K}^{-} \backslash G\right]$ belong to $\mathcal{S}_{J}$. Conjecture 19.1 would imply that they also belong to $\mathcal{B}_{J}$. In particular, when $\widetilde{\mathcal{A}}_{J}$ has finite cluster type, $\mathcal{B}_{J}$ should be equal to the set of cluster monomials.

Regarding total positivity, we propose the following conjecture, inspired by Fomin and Zelevinsky's approach to total positivity via cluster algebras. Let $X$ denote the partial flag variety $B_{K}^{-} \backslash G$ and let $X_{>0}$ be the totally positive part of $X$ [30]. Lusztig has shown that it can be defined by $\operatorname{dim} L(\lambda)$ algebraic inequalities given by the elements of $\mathcal{B}(\lambda)$ for a "sufficiently large" $\lambda \in \Pi_{J}$ [30, Th. 3.4]. In fact $X_{>0} \subset N_{K}$, where $N_{K}$ is embedded in $X$ as in $\S 17$. We propose the following alternative descriptions of $X_{>0}$ by systems of $d=\operatorname{dim} X$ algebraic inequalities.

Conjecture 19.2. Let $T=T_{1} \oplus \cdots \oplus T_{d}$ be a basic complete rigid $\Lambda$-module in $\operatorname{Sub} Q_{J}$. Then $x \in N_{K}$ belongs to $X_{>0}$ if and only if

$$
\varphi_{T_{i}}(x)>0, \quad(i=1, \ldots, d) .
$$

Example 19.3. We consider again type $D_{4}$ and $J=\{4\}$, so that $X$ can be identified with the Grassmannian of isotropic lines in $\mathbb{C}^{8}$, as in $\S 2$. In this case $\Pi_{J}=\mathbb{N} \varpi_{4}$ and Lusztig's description involves $\operatorname{dim} L(\lambda)$ inequalities where $\lambda=k \varpi_{4}$ with $k \geq 5$. For example $\operatorname{dim} L\left(5 \varpi_{4}\right)=672$.

On the other hand the category $\operatorname{Sub} Q_{4}$ has 4 basic complete rigid modules:

$$
\begin{array}{ll}
M_{4} \oplus M_{5} \oplus M_{6} \oplus M_{7} \oplus M_{8} \oplus Q_{4}, \quad M_{4} \oplus M_{5} \oplus M_{6} \oplus M_{7}^{*} \oplus M_{8} \oplus Q_{4}, \\
M_{4} \oplus M_{5} \oplus M_{6} \oplus M_{7} \oplus M_{8}^{*} \oplus Q_{4}, \quad M_{4} \oplus M_{5} \oplus M_{6} \oplus M_{7}^{*} \oplus M_{8}^{*} \oplus Q_{4},
\end{array}
$$

where we have used the notation of Examples 13.3 and 16.2. Each of them gives rise to a positivity criterion consisting of $\operatorname{dim} X=6$ inequalities. Using the notation of $\S 2$, these are respectively

$$
\begin{aligned}
& y_{4}>0, p>0, y_{5}>0, y_{2}>0, y_{3}>0, y_{8}>0 \\
& y_{4}>0, p>0, y_{5}>0, y_{7}>0, y_{3}>0, y_{8}>0 ; \\
& y_{4}>0, p>0, y_{5}>0, y_{2}>0, y_{6}>0, y_{8}>0 ; \\
& y_{4}>0, p>0, y_{5}>0, y_{7}>0, y_{6}>0, y_{8}>0
\end{aligned}
$$

Note that since we regard $X_{>0}$ as a subset of $N_{K}$, the additional relation $y_{1}=1$ is understood. Thus the conjecture holds in this case, and more generally in type $D_{n}$ when $J=\{n\}$.

When $X=B^{-} \backslash G$ and $M$ is a rigid module in $\mathcal{R}$, the conjecture follows from the work of Berenstein, Fomin and Zelevinsky and our construction. When $X$ is a type $A$ Grassmannian and $M$ is a rigid module in $\mathcal{R}$, the conjecture follows from the work of Scott [34] and our construction. 


\section{References}

[1] E. Abe, Hopf Algebras. Cambridge Tracts in Mathematics 74, Cambridge University Press, Cambridge, 1980.

[2] M. Auslander and S. Smalø, Almost split sequences in subcategories, J. Algebra 69 (1981), 426-454.

[3] A. Berenstein, S. Fomin and A. Zelevinsky, Cluster algebras. III. Upper bounds and double Bruhat cells, Duke Math. J. 126 (2005), 1-52.

[4] A. B. Buan, R. Marsh, M. Reineke, I. Reiten and G. Todorov, Tilting theory and cluster combinatorics, Advances Math. 204 (2006), 572-618.

[5] P. Caldero, B. Keller, ¿From triangulated categories to cluster algebras, arXiv:math.RT/0506018, to appear in Invent. Math.

[6] R. Carter, G. Segal and I. Macdonald, Lectures on Lie Groups and Lie Algebras. London Mathematical Society Student Texts 32, Cambridge University Press, Cambridge, 1995.

[7] V. Dlab and C.M. Ringel, The module theoretical approach to quasi-hereditary algebras. In Representations of Algebras and Related Topics. London Mathematical Society Lecture Note Series 168, Cambridge Univ. Press, Cambridge, 1992, 200-224.

[8] W. Fulton and J. Harris, Representation Theory. A First Course. Graduate Texts in Mathematics 129. Readings in Mathematics. Springer-Verlag, New York-Heidelberg, 1991.

[9] S. Fomin and A. Zelevinsky, Double Bruhat cells and total positivity, J. Amer. Math. Soc. 12 (1999), 335-380.

[10] S. Fomin and A. Zelevinsky, Cluster Algebras. I. Foundations, J. Amer. Math. Soc. 15 (2002), 497-529.

[11] S. Fomin and A. Zelevinsky, Cluster Algebras. II. Finite type classification, Invent. Math. 154 (2003), 63-121.

[12] C. Geiss, B. Leclerc and J. Schröer, Semicanonical bases and preprojective algebras, Ann. Sci. École Norm. Sup. 38 (2005), 193-253.

[13] C. Geiss, B. Leclerc and J. Schröer, Verma modules and preprojective algebras, Nagoya Math. J. 182 (2006), 241-258.

[14] C. Geiss, B. Leclerc and J. Schröer, Rigid modules over preprojective algebras, Invent. Math. 165 (2006), 589-632.

[15] C. Geiss, B. Leclerc and Jan Schröer, Auslander algebras and initial seeds for cluster algebras, J. London Math. Soc. 75 (2007), 718-740.

[16] C. Geiss, B. Leclerc and Jan Schröer, Semicanonical bases and preprojective algebras II: a multiplication formula, Compositio Math. 143 (2007), 1313-1334.

[17] C. Geiss, B. Leclerc and Jan Schröer, Partial flag varieties and preprojective algebras, Ann. Inst. Fourier, to appear.

[18] C. Geiss, B. Leclerc and Jan Schröer, Cluster algebra structures and semicanonical bases for unipotent groups, in preparation.

[19] C. Geiß and J. Schröer, Extension-orthogonal components of preprojective varieties, Trans. Amer. Math. Soc. 357 (2005), 1953-1962. 
[20] I.M. Gelfand and V.A. Ponomarev, Model algebras and representations of graphs, Funktsional. Anal. i Prilozhen. 13 (1979), 1-12.

[21] J. E. Humphreys, Linear Algebraic Groups. Graduate Texts in Mathematics 21, Springer-Verlag, New York-Heidelberg, 1975.

[22] M. Kashiwara, On crystal bases of the $Q$-analogue of universal enveloping algebras, Duke Math. J. 63 (1991), 465-516.

[23] B. Keller, I. Reiten, Acyclic Calabi-Yau categories, arXiv:math/0610594.

[24] V. Lakshmibai and N. Gonciulea, Flag Varieties. Travaux en cours 63, Hermann, Paris, 2001.

[25] B. Leclerc, Imaginary vectors in the dual canonical basis of $U_{q}(\mathfrak{n})$, Transform. Groups 8 (2003), 95-104.

[26] G. Lusztig, Canonical bases arising from quantized enveloping algebras, J. Amer. Math. Soc. 3 (1990), 447-498.

[27] G. Lusztig, Quivers, perverse sheaves, and quantized enveloping algebras, J. Amer. Math. Soc. 4 (1991), 365-421.

[28] G. Lusztig, Introduction to quantized enveloping algebras. In New Developments in Lie Theory and their Applications. Progress in Mathematics 105, Birkhäuser-Verlag, Basel-Boston, 1992, 49-65.

[29] G. Lusztig, Total positivity in reductive groups. In Lie Theory and Geometry. Progress in Mathematics 123, Birkhäuser-Verlag, Basel-Boston, 1994, 531-568.

[30] G. Lusztig, Total positivity in partial flag manifolds, Represent. Theory 2 (1998), $70-78$.

[31] G. Lusztig, Introduction to total positivity. In Positivity in Lie Theory: Open Problems. de Gruyter Expositions in Mathematics 26, Walter de Gruyter, Berlin-New York, 1998, 133-145.

[32] G. Lusztig, Semicanonical bases arising from enveloping algebras, Advances Math. 151 (2000), 129-139.

[33] R. Marsh, M. Reineke and A. Zelevinsky, Generalized associahedra via quiver representations, Trans. Amer. Math. Soc. 355 (2003), 4171-4186.

[34] J. S. Scott, Grassmannians and cluster algebras, Proc. London Math. Soc. 92 (2006), $345-380$.

Christof Geiss, Instituto de Matemáticas, Universidad Nacional Autónoma de México, 04510 México D.F., México

E-mail: christof@math.unam.mx

Bernard Leclerc, Université de Caen, LMNO UMR 6139, F-14032 Caen cedex, France E-mail: leclerc@math.unicaen.fr

Jan Schröer, Mathematisches Institut, Universität Bonn, Beringstr. 1, D-53115 Bonn, Germany

E-mail: schroer@math.uni-bonn.de 